\title{
Quantum chaotic scattering in time-dependent external fields: random matrix approach
}

\author{
Maxim G. Vavilov \\ Department of Applied Physics, Yale University, New Haven, CT 06520 \\ E-mail: mv44@cornell.edu
}

\begin{abstract}
We review the random matrix description of electron transport through open quantum dots, subject to time-dependent perturbations. All characteristics of the current linear in the bias can be expressed in terms of the scattering matrix, calculated for a time-dependent Hamiltonian. Assuming that the Hamiltonian belongs to a Gaussian ensemble of random matrices, we investigate various statistical properties of the direct current in the ensemble. Particularly, even at zero bias the time-dependent perturbation induces current, called photovoltaic current. We discuss dependence of the photovoltaic current and its noise on the frequency and the strength of the perturbation. We also describe the effect of time-dependent perturbation on the weak localization correction to the conductance and on conductance fluctuations.
\end{abstract}

PACS numbers: 73.23.Ad, 72.15.Rn, 72.70.+m 


\section{Introduction}

Quantum dot is a small disordered or irregularly shaped conductor, connected to leads [1, see Fig. 1. Exact values of the conductance of a quantum dot are determined by electron wave functions in the system and are hard to calculate exactly for arbitrary configurations of the dot. Moreover, the conductance changes significantly even for tiny changes in the position of impurities or the boundary of the dot. Due to extreme sensitivity of the conductance on many parameters, the statistical description of the conductance is more appropriate [2, 3, 4, 5, 6, 7, 8, 9, 10, The random fluctuations of the conductance from sample to sample of non-interacting systems are universal. The universality 11, 12, means that the conductance statistics can be described by universal functions, which are independent from the shape of the dot or the details of the disordered potential. Particularly, the variance of the conductance varg is of the order $G_{0}^{2}$ and is nearly independent from the sample geometry $\left(G_{0}=e^{2} / \pi \hbar\right.$ is the quantum of conductance for spin degenerate electrons). The other universal quantity is the weak localization correction to the conductance, defined as the difference of average values of the conductance over orthogonal (zero magnetic field) and unitary (strong magnetic field) ensembles. The weak localization correction to the conductance is also of the order of $G_{0}$ [13, 14, 15, 16, 17, 18, 19].

A common description of electron transport through quantum dots is based on the Landauer formalism 20, 21, 22, 23, when the transport characteristics of the system are described in terms of the scattering amplitudes between different conducting channels in the leads. There are several approaches for statistical description of electron transport. One approach is based on a diagram technique developed for disordered bulk metals [24, when the scattering amplitudes are represented in terms of electron Green functions [4, 25, 26, 27.

Alternative approaches are based on the description of the system by random matrices, when either an exact scattering matrix is replaced by a random unitary matrix, or an exact Hamiltonian is replaced by a random Hermitian matrix. In the first case, the unitary matrix is taken from Dyson's circular ensemble of uniformly distributed random matrices 28, 18, 19. In the Hamiltonian approach, the Hermitian matrix belongs to an ensemble of random matrices [29] with the Gaussian distribution of its matrix elements 30, 31. The equivalence for statistical description of electron transport by both random matrix approaches was shown in 31, 32, 33.

Although the random matrix approach is not based on microscopic description of electron system, their correspondence to microscopic problem has been proven for disordered metal grains [34, 35, 36]. The validity of such random matrix description of chaotic ballistic systems was addressed in 37, 38, 12.

We imply the following realization of the system, see Fig. 1 a). Negative voltages applied to the gates (black areas) confine electrons to a small region (light gray), forming a quantum dot. Electrons in the dot are connected to the electron reservoirs by narrow leads. Electric current that flows through the dot can be measured as a function of the voltage bias $V$ between the reservoirs and the amplitudes of ac gate voltages $V_{1,2}(t)$. Particularly, the current linear in bias $V$ is determined by the conductance of the dot. Changing magnetic field or shape of the dot one can obtain different realizations of the quantum dot and experimentally study statistics of the quantum corrections to the conductance.

The quantum corrections to the conductance are commonly characterized by the weak localization and the variance of conductance fluctuations. As any other quantum 
interference phenomena, they are very sensitive to inelastic processes, commonly referred to as dephasing 39. A phenomenological description of the effect of dephasing on electron transport through open quantum dots was developed in Refs. 40, 41, 42. The dephasing rate due to electron-electron interaction in quantum dots was estimated in 43 44]. Another possible source of dephasing is a time-dependent perturbation, such as a microwave radiation or periodic deformation. In this case the Hamiltonian of the system can be considered as a time-dependent random matrix [45, 46, 47], and all transport quantities can be calculated as a function of various parameters (e.g. strength and frequency) of the time-dependent perturbation. The scattering matrix description of the system subject to time-dependent perturbation was developed in energy representation by Büttiker, Thomas and Pretrein in Refs. [8, 49]. In this case the scattering matrix describes processes when electron scattering between different channels in the leads is accompanied by the change of electron energy. Alternatively, the analysis of the effect of time-dependent perturbation on the conductance can be carried out in time representation, see Refs. 45, 46, 47, 50, 51]. In general, both the weak localization correction to the conductance and the variance of conductance fluctuations are suppressed by time-dependent perturbation. The suppression of the quantum corrections to the conductance by microwave radiation was observed experimentally in [10].

Time-dependent perturbation of quantum dots not only suppresses quantum corrections to the conductance, but also produces electric current through the system even at zero bias. This effect is related to the charge pumping, which occurs in systems with large tunnel barriers [52, 53, 54, 55]. If the conductance of the system is very small, the electric current is quantized in units of $e \omega / 2 \pi$, where $2 \pi / \omega$ is the period of the pump. At finite conductance, a countercurrent reduces the pumped current and thus violate the quantization of electric current [56]. For an open quantum dot, the countercurrent nearly compensates the pumped current and the current is no longer quantized.

In the low frequency limit, the magnitude of the pumped current is determined entirely by the evolution of the system in the parameter space, see Fig. $1 \mathrm{~b}$ ), under time dependent perturbations [57, 58, 59, 60]. As frequency increases, the parametric description becomes insufficient and requires full analysis of electron dynamics in timedependent fields [61, 47]. The analysis of how the adiabatic description breaks down at finite frequency can be also found in Refs. 62, 63. We note that the charge pumping through an open quantum dot is a manifestation of the photovoltaic effect, which occurs in systems without inversion center [64. The photovoltaic effect was previously considered by Falko and Khmelnitskii [65] in mesoscopic microjunctions and by Kravtsov, Aronov and Yudson [66, 67, in normal metal rings.

It turns out 61 that the photovoltaic current is sensitive to the actual electron distribution function in the dot. Time-dependent perturbations may broaden the distribution function, resulting in heating. This broadening of the electron distribution occurs as a result the electron diffusion in the energy space. The effect of time-dependent perturbations on electron distribution function becomes even more interesting in closed systems, when the energy diffusion acquires quantum interference corrections. The latter leads to a dynamic localization 68] of the electrons in energy space 69, 70].

Photovoltaic current fluctuates not only with respect to different realizations of the quantum dot, but also for a given realization due to quantum and thermal fluctuations. Such fluctuations are called current noise and are described by the 
a)

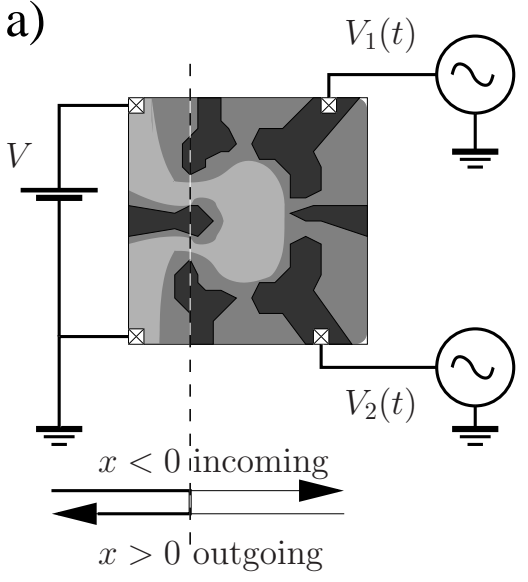

b)

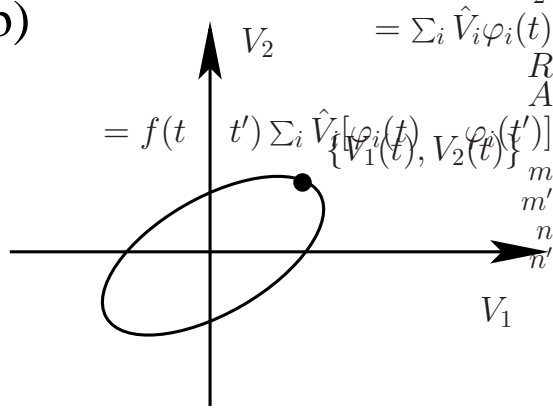

Figure 1. a) Schematic picture of the experimental setup. Light gray color shows the region available for free electron motion, while the dark gray color shows the region forbidden for electrons due to electrostatic repulsion from the gates (shown in black) with applied negative voltages. A finite bias $V$ is applied between the upper and lower (in the text refereed to as left and right) reservoirs. Oscillating voltages $V_{1,2}(t)$ applied to the gates produce time-dependent perturbation of the electron system. b) Contour plot represents time evolution of gate voltages $V_{1,2}(t)$.

fluctuations of the charge transported through the dot in a certain number of perturbation cycles. The statistics of such charge fluctuations was studied in Refs. [71, 72, 73, 74, 75, 76, for temperatures $T$ and pumping frequencies $\omega$ much smaller than the inverse dwell time $\gamma_{\text {esc }}$ (escape rate) of electrons from the quantum dot. Particularly, Refs. [71, 72] addressed the full counting statistics at temperatures $T \ll \omega$ (we use $\hbar=1$ and the Boltsmann constant $k_{\mathrm{B}}=1$ ). The mean square charge fluctuations for $\omega, T \ll \gamma_{\text {esc }}$ (but for arbitrary relation between $\omega$ and $T$ ) were considered in Ref. 76. The variance of the photovoltaic current for arbitrary relation between the temperature $T$, the frequency $\omega$, the escape rate $\gamma_{\text {esc }}$ and the strength of the perturbation was calculated in Ref. [77].

Experiments 78, 79, 80 were performed to detect the photovoltaic current in various mesoscopic systems [78, 79, including open quantum dots [80] in the adiabatic regime. The observed magnetic field symmetry and the amplitude of the current indicate that the measured current was likely related to the ac rectification [81, 82, 83]. A more detailed analysis of the zero-bias current in different regimes of microwave radiation shows that in some instances the photovoltaic current, and not the rectification current, was observed 82] 83].

In this review we focus on the random matrix description of electron transport through open quantum dots in the limit of the large number of open channels $N_{\mathrm{ch}}$ connecting the dot to the leads. This condition allows us to neglect the electronelectron interaction that gives corrections of the $1 / N_{\mathrm{ch}}^{2}$ order, see Ref. [84]. The same condition permits the use of a diagrammatic technique, similar to that described in 24, to calculate ensemble averaging. We assume that the electron dynamics in the dot is fully chaotic and disregard classical fluctuations of the conductance [26]. We emphasize that the random matrix description is applicable for sufficiently small quantum dots, when the Thouless energy $E_{\mathrm{T}}=1 / \tau_{\text {cross }}$ is much greater than all 
other energy scales of the problem, such as the frequency $\omega$ of the perturbation or the temperature $T$ ( $\tau_{\text {cross }}$ is the electron crossing time of the dot). Larger systems $\left(E_{\mathrm{T}} \ll \omega, T\right)$ can be treated by methods developed for bulk conductors [39, see e.g. 50. We note that the derivation of the results will be performed within the Hamiltonian formalism, following Refs. 45, 46, 61, but the same results were derived within scattering matrix formalism in [47.

\section{Scattering Matrix Formulation of Transport through Open Quantum Dots}

\subsection{Model}

The Hamiltonian of the system is

$\hat{\mathcal{H}}=\hat{\mathcal{H}}_{\mathrm{d}}+\hat{\mathcal{H}}_{\mathrm{ld}}+\hat{\mathcal{H}}_{\mathrm{l}}$.

We choose the basis for electron wave functions in the dot, so that the coupling of states in the dot to states in the leads can be written as

$\hat{H}_{\mathrm{ld}}=\sum_{\alpha, n, k}\left(W_{n \alpha} \psi_{\alpha}^{\dagger}(k) \psi_{n}+\right.$ H.c. $), \quad W_{n \alpha}= \begin{cases}\mathcal{T}, & \text { if } n=\alpha \leq N_{\mathrm{ch}}, \\ 0, & \text { otherwise. }\end{cases}$

Here $\psi_{n}$ and $\psi_{\alpha}(k)$ are the annihilation operators of electrons in the dot and the leads, respectively. Index $n$ enumerates electron states in the dot: $n=1, \ldots M$, with $M \rightarrow \infty$. Index $\alpha$ labels channels in the leads, with $1 \leq \alpha \leq N_{\mathrm{l}}$ for the $N_{\mathrm{l}}$ channels in the left lead and with $N_{\mathrm{l}}+1 \leq \alpha \leq N_{\mathrm{ch}}$ for the $N_{\mathrm{r}}$ channels in the right lead, $N_{\mathrm{ch}}=N_{\mathrm{l}}+N_{\mathrm{r}}$. Coupling constants $\mathcal{T}$ are defined below in Eq. (43). The Hamiltonian for electron states in the leads near the Fermi surface can be linearized:

$\hat{H}_{\mathrm{l}}=v_{\mathrm{F}} \sum_{\alpha, k} k \psi_{\alpha}^{\dagger}(k) \psi_{\alpha}(k)$,

where the continuous variable $k$ denotes electron momenta in the leads, $v_{\mathrm{F}}=(2 \pi \nu)^{-1}$ is the Fermi velocity, and $\nu$ is the density of states per channel at the Fermi surface.

Finally, $\hat{\mathcal{H}}_{\mathrm{d}}$ is the Hamiltonian of the electrons in the dot, determined by the $M \times M$ matrix $\hat{H}$ and the electrostatic energy of $N$ electrons:

$\mathcal{H}_{\mathrm{d}}=\boldsymbol{\psi}^{\dagger}\left[\hat{H}+\sum_{i}\left(\hat{V}_{i}+\hat{1} Z_{i}\right) \varphi_{i}(t)\right] \boldsymbol{\psi}+E_{\mathrm{c}} N^{2}$.

Matrix $\hat{H}$ describes the time independent part of the electron Hamiltonian, and the time-dependent component of the Hamiltonian is represented in terms of the traceless matrices $\hat{V}_{i}$ and the diagonal matrix $\hat{1} Z_{i}$. In the setup shown in Fig. 1, the timedependent perturbation is generated by the gate voltages $V_{1,2}(t)$. The perturbation is linear in small amplitude of oscillating voltages $V_{1,2}(t)$, and the time evolution of the perturbation characterized by the dimensionless functions $\varphi_{i}(t) \propto V_{i}(t)$. The second term in Eq. (4) represents the largest in $1 / M$ contribution from the electron-electron interaction with $E_{\mathrm{c}}$ being the charging energy of the dot, and $N=\sum_{n} \psi_{n}^{\dagger} \psi_{n}$ being the operator of the electron number in the dot. The status of this approximation was discussed in detail in Ref. [85]. For an open quantum dot with the large number of open channels $N_{\mathrm{ch}} \gg 1$ the interaction term can be treated within mean field approximation, and the Hamiltonian $\hat{\mathcal{H}}_{\mathrm{d}}$ in Eq. (4) can be further simplified:

$\mathcal{H}_{\mathrm{d}}=\boldsymbol{\psi}^{\dagger}\left[\hat{H}+\sum_{i} \hat{V}_{i} \varphi_{i}(t)+\hat{1} e V_{\mathrm{d}}(t)\right] \boldsymbol{\psi}, \quad e V_{\mathrm{d}}(t)=\sum_{i} Z_{i} \varphi_{i}(t)+2 E_{\mathrm{c}}\langle N\rangle$. 
Here we introduced the electric potential $V_{\mathrm{d}}(t)$ linear in the quantum mechanical average $\langle N\rangle$ of the electron number $N$ in the dot. Corrections to this mean field treatment were calculated in Refs. [84, 86, 87,

To determine the electric potential $V_{\mathrm{d}}(t)$ in the dot, we have to define the quantum mechanical average $\langle N\rangle$ of the electron number $N$ in the dot. In each particular moment of time the electron number $\langle N\rangle$ is not constant and its time evolution is described by the discontinuity equation $e \dot{N}(t)=I_{\mathrm{r}}(t)+I_{1}(t)$. We estimate $V_{\mathrm{d}}(t)$ to the lowest order in $1 / N_{\mathrm{ch}} \ll 1$, and use $N_{\mathrm{l}} G_{0}\left(N_{\mathrm{r}} G_{0}\right)$ for the conductance of the left (right) contact, $G_{0}=e^{2} / \pi$ is the quantum conductance. Then, $\langle N(t)\rangle$ satisfies the following equation

$\frac{d\langle N(t)\rangle}{d t}=-\gamma_{\mathrm{esc}}\langle N(t)\rangle+\frac{e N_{\mathrm{l}}}{\pi}\left(V_{\mathrm{l}}-V_{\mathrm{d}}(t)\right)+\frac{e N_{\mathrm{r}}}{\pi}\left(V_{\mathrm{r}}-V_{\mathrm{d}}(t)\right)$.

The first term in Eq. (6) is a diffusion term, describing the electron escape from the dot with rate $\gamma_{\text {esc }}$, where $\gamma_{\text {esc }}^{-1}$ is the mean time for an electron to escape the dot through one of the leads; below we define $\gamma_{\text {esc }}$ in terms of microscopic parameters of the system. The last two terms in Eq. (6) represent electron flux from the dot due to the voltage difference $V_{\mathrm{l},(\mathrm{r})}-V_{\mathrm{d}}(t)$ across the contact of the left (right) reservoir and the dot. A discussion of the charge dynamics in quantum dots can be also found in 88 .

Combining Eq. (6) with the expression for $V_{\mathrm{d}}(t)$ from Eq. (51), we obtain

$V_{\mathrm{d}}(t)=\frac{2 E_{\mathrm{c}} N_{\mathrm{ch}}}{2 E_{\mathrm{c}} N_{\mathrm{ch}}+\pi \gamma_{\mathrm{esc}}} \frac{N_{\mathrm{l}} V_{\mathrm{l}}+N_{\mathrm{r}} V_{\mathrm{r}}}{N_{\mathrm{ch}}}+\frac{\gamma_{\mathrm{esc}}+\partial_{t}}{\gamma_{\mathrm{esc}}+2 E_{\mathrm{c}} N_{\mathrm{ch}} / \pi+\partial_{t}} \sum Z_{i} \varphi_{i}(t)$.

The characteristic energy scale governing the dynamics of the charge is $E_{\mathrm{c}} N_{\mathrm{ch}} / 2 \pi \propto$ $G_{0} N_{\mathrm{ch}} / C_{\mathrm{d}}, C_{\mathrm{d}}$ is the dot capacitance. Usually, this scale is of the order of the Thouless energy $E_{T}$ and significantly exceeds electron escape rate $\gamma_{\text {esc }}$. Therefore, we consider the limit, when both $\gamma_{\text {esc }}$ and the frequency of the external field $\omega$ are much smaller than $E_{\mathrm{c}}$, and use the following equation for the electrostatic potential of the dot

$V_{\mathrm{d}}(t) \equiv V_{\mathrm{d}}=\frac{N_{\mathrm{l}} V_{\mathrm{l}}+N_{\mathrm{r}} V_{\mathrm{r}}}{N_{\mathrm{ch}}}$.

We conclude that the time-dependent perturbation Eq. (4) can be chosen traceless, $Z_{i}=0$, and the electric potential in the middle dot is determined by the potentials of the left and right reservoirs.

\subsection{Electric Current}

The current through the dot is given in terms of the scattering matrices $\hat{\mathcal{S}}\left(t, t^{\prime}\right)$ by the following expression

$\langle I\rangle=e \int_{0}^{\tau_{\mathrm{o}}} \frac{d t}{\tau_{\mathrm{o}}} \int d t_{1} d t_{2} \operatorname{tr}\left\{\hat{\Lambda}\left[\hat{\mathcal{S}}\left(t, t_{1}\right) \hat{f}\left(t_{1}-t_{2}\right) \hat{\mathcal{S}}^{\dagger}\left(t_{2}, t\right)-\hat{f}(+0)\right]\right\}$.

The derivation of Eq. (9) can be found in [49, 61, 47, see also Appendix A Here $\langle I\rangle$ stands for the quantum mechanical and thermodynamic averages of the current operator (no ensemble averaging!) and $\hat{f}(t)$ represents the electron distribution function in the leads in time representation. We consider the case when electrons in the leads are in thermal equilibrium at temperature $T$, but the different voltages $V_{1}$ and $V_{\mathrm{r}}$ are applied to the left and right electron reservoirs. Then, the matrix $\hat{f}(t)$ is 
diagonal $f_{\alpha \alpha}(t)=f_{1(\mathrm{r})}(t)$, if channel $\alpha$ belongs to the left (right) lead. The function $f_{1(\mathrm{r})}(t)$ is the Fourier transform of the Fermi-Dirac distribution function:

$f_{1(\mathrm{r})}(\tau)=e^{i e V_{1(\mathrm{r})}} f(\tau) ; \quad f(\tau)=\int_{-\infty}^{+\infty} \frac{d \omega}{2 \pi} e^{i \omega \tau}\left\{\frac{1}{e^{\omega / T}+1}-\frac{1}{2}\right\}=\frac{i T}{2 \sinh \pi T \tau}$.

Here the traceless diagonal matrix $\hat{\Lambda}$ is introduced

$\Lambda_{\alpha \beta}=\delta_{\alpha \beta} \begin{cases}+\frac{N_{\mathrm{r}}}{N_{\mathrm{ch}}}, & \text { if } 1 \leq \alpha \leq N_{\mathrm{l}} \\ -\frac{N_{\mathrm{l}}}{N_{\mathrm{ch}}}, & \text { if } N_{\mathrm{l}}<\alpha \leq N_{\mathrm{ch}},\end{cases}$

and the scattering matrix $\hat{\mathcal{S}}\left(t, t^{\prime}\right)$

$\mathcal{S}_{\alpha \beta}\left(t, t^{\prime}\right)=e^{i e V_{\mathrm{d}}\left(t-t^{\prime}\right)}\left[\delta_{\alpha \beta} \delta\left(t-t^{\prime}\right)-2 \pi i \nu W_{\alpha n}^{\dagger} G_{n m}^{R}\left(t, t^{\prime}\right) W_{m \beta}\right]$,

is defined in terms of the Green function $G_{n m}^{R}\left(t, t^{\prime}\right)$ that satisfies the following equation

$$
\left(i \frac{\partial}{\partial t}-\hat{H}-\sum_{i} \hat{V}_{i} \varphi_{i}(t)+i \pi \nu \hat{W} \hat{W}^{\dagger}\right) \hat{G}^{(R)}\left(t, t^{\prime}\right)=\delta\left(t-t^{\prime}\right),
$$

where the matrices $\hat{H}, \hat{V}_{i}$ and $\hat{W}$ were introduced earlier, see Eqs. (2) and (5). The diagonal component $e V_{\mathrm{d}}$ of the electron Hamiltonian in the dot is removed from the expression for the electron Green function $G_{n m}^{R}\left(t, t^{\prime}\right)$ by the gauge transformation, represented by the exponential factor in Eq. (12).

To the linear order in voltage across the dot $V=V_{1}-V_{\mathrm{r}}$, the dc electric current has the form

$\langle I\rangle=I_{\mathrm{ph}}+g V$.

The first term represents the photovoltaic current, which flows through the dot even at zero bias. The second term is linear in voltage $V$ with factor $g$ being the dc conductance of the dot in the presence of time-dependent perturbations $\hat{V}_{i}$. The linear in $V$ contribution to the current in general may come from two sources: i) the non-equilibrium distribution of electrons in the leads and ii) change in the photovoltaic current $I_{\mathrm{ph}}$ due to change in the configuration of the electron wave functions when the bias is applied. Due to the electro-neutrality condition Eq. (7), the voltages $V_{l}$, $V_{\mathrm{d}}$ and $V_{\mathrm{d}}$ enter only as exponential factors to the expression for the electric current Eq. (9), and do not actually affect the structure of electron wave functions in the dot. Therefore, only the non-equilibrium current contributes to the linear in $V$ term.

The dc conductance $g$ of the dot can be represented in the form

$g=g_{\mathrm{cl}}+G_{0} \int_{0}^{\tau_{\mathrm{o}}} \frac{d t}{\tau_{\mathrm{o}}} \int_{-\infty}^{+\infty} d t_{1} d t_{2} F\left(t_{1}-t_{2}\right) \operatorname{tr}\left\{\hat{\mathcal{S}}\left(t, t_{1}\right) \hat{\Lambda} \mathcal{S}^{\dagger}\left(t_{2}, t\right) \hat{\Lambda}\right\}, \quad g_{\mathrm{cl}}=G_{0} \frac{N_{\mathrm{l}} N_{\mathrm{r}}}{N_{\mathrm{ch}}}$.

Here $g_{\mathrm{cl}}$ is the classical conductance of the dot, $\tau_{\mathrm{o}}$ is the observation time, $G_{0}=e^{2} / \pi \hbar$ is the quantum conductance for doubly degenerate electrons in spin states and $F(x)$ is the Fourier transform of the derivative of electron distribution function:

$F(t)=\frac{\pi T t}{\sinh \pi T t}$. 
The expression for the photovoltaic current $I_{\mathrm{ph}}$ can be obtained from Eq. (9) by taking $V_{1}=V_{\mathrm{r}}$. Using the Wigner transform for the scattering matrix

$$
\hat{\mathcal{S}}\left(t, t^{\prime}\right)=\int \hat{\mathcal{S}}_{\frac{t+t^{\prime}}{2}}(\varepsilon) e^{i \varepsilon\left(t-t^{\prime}\right)} \frac{d \varepsilon}{2 \pi},
$$

we write

$I_{\mathrm{ph}}=e \int_{0}^{\tau_{\mathrm{o}}} \frac{d t}{\tau_{\mathrm{o}}} \int d \tau \int \frac{d \varepsilon}{2 \pi} e^{i \varepsilon \tau} f(\tau) \operatorname{tr}\left\{\hat{\Lambda} \hat{\mathcal{S}}_{\frac{t}{2}+\frac{\tau}{4}}(\varepsilon) \hat{\mathcal{S}}_{\frac{t}{2}-\frac{\tau}{4}}^{\dagger}(\varepsilon)\right\}$.

For slow perturbations $\varphi_{i}$ with frequencies $\omega_{i}$ smaller than temperature $T$ or the inverse eigenvalues of the time delay matrix 89

$\hat{\mathcal{R}}_{\varepsilon}(\varepsilon, t)=\left[\partial_{\varepsilon} \hat{\mathcal{S}}_{t}(\varepsilon)\right] \hat{\mathcal{S}}_{t}^{\dagger}(\varepsilon)$,

we can expand the scattering matrices in Eq. (18) in $\tau$ and obtain

$I_{\mathrm{ph}}=e \int_{0}^{\tau_{\mathrm{o}}} \frac{d t}{\tau_{\mathrm{o}}} \int \frac{d \varepsilon}{2 \pi} \frac{1}{\cosh ^{2} \varepsilon / 2 T} \operatorname{tr}\left\{\hat{\Lambda}\left(\frac{\partial \mathcal{S}_{t}(\varepsilon)}{\partial t} \mathcal{S}_{t}^{\dagger}(\varepsilon)-\mathcal{S}_{t}(\varepsilon) \frac{\partial \mathcal{S}_{t}^{\dagger}(\varepsilon)}{\partial t}\right)\right\}$.

Here, the scattering matrix in the Wigner representation is a function of the perturbation itself and its time derivatives: $\hat{\mathcal{S}}_{t}(\varepsilon)=\hat{\mathcal{S}}\left(\varepsilon, \varphi_{i}(t), \dot{\varphi}_{i}(t), \ldots\right)$ because the Green function $\hat{G}^{(R)}(\varepsilon, t)$ is a solution of the equation:

$$
\begin{aligned}
\varepsilon \hat{G}(\varepsilon, t) & -\frac{1}{2}\left\{\hat{H}-i \pi \nu \hat{W} \hat{W}^{\dagger} ; \hat{G}^{(R)}(\varepsilon, t)\right\} \\
& +\sum_{k=0}^{\infty} \sum_{i} \frac{1}{2(2 i)^{k} k !} \frac{d^{k} \varphi_{i}(t)}{d t^{k}}\left(\hat{V}_{i} \frac{\partial^{k} \hat{G}(\varepsilon, t)}{\partial \varepsilon^{k}}+(-1)^{k} \frac{\partial^{k} \hat{G}(\varepsilon, t)}{\partial \varepsilon^{k}} \hat{V}_{i}\right)=1,
\end{aligned}
$$

with $\{\hat{A} ; \hat{B}\}=\hat{A} \hat{B}+\hat{B} \hat{A}$. In the adiabatic approximation the derivatives $d^{k} \varphi_{i}(t) / d t^{k}$ can be neglected and the scattering matrix is determined by parameters $\varphi_{i}(t)$, $\hat{\mathcal{S}}_{t}(\varepsilon)=\hat{\mathcal{S}}\left(\varepsilon, \varphi_{i}(t)\right)$ :

$I_{\mathrm{ph}}=\frac{e}{T_{\mathrm{p}}} \oint d \varphi_{i} \int \frac{\operatorname{tr}\left\{\hat{\Lambda} \operatorname{Im} \hat{\mathcal{R}}_{i}(\varepsilon, \varphi)\right\}}{\cosh ^{2} \varepsilon / 2 T} \frac{d \varepsilon}{2 \pi}, \quad \hat{\mathcal{R}}_{i}(\varepsilon, \varphi)=\frac{\partial \hat{\mathcal{S}}(\varepsilon, \varphi)}{\partial \varphi_{i}} \hat{\mathcal{S}}^{\dagger}(\varepsilon, \varphi)$.

The integral in Eq. (22) runs over the loop in the parameter space $\varphi_{i}$, and $T_{\mathrm{p}}$ is the time for a system to complete this loop. Particularly, for the perturbation characterized by two parameters

$\varphi_{1}(t)=X_{1} \cos (\omega t), \varphi_{2}(t)=X_{2} \cos (\omega t+\phi)$

the photovoltaic current is given by [57.

$I_{\mathrm{ph}}=\frac{e \omega}{2 \pi^{2}} \int_{A} d \varphi_{1} d \varphi_{2} \operatorname{Im} \operatorname{tr}\left\{\hat{\Lambda} \frac{\partial \hat{\mathcal{S}}}{\partial \varphi_{1}} \frac{\partial \hat{\mathcal{S}}^{\dagger}}{\partial \varphi_{2}}\right\}$

where the integral runs over the inner part of the ellipse, defined by Eq. (23). At finite frequencies, but still $\omega_{i} \ll T$, Eq. (20) is still applicable and can be rewritten in the form similar to Eq. (22), if the parameter space $\varphi_{i}$ is extended to the phase space, containing time derivatives of $\varphi_{i}(t)$ as well.

With the help of the equations of motion Eq. (13), the expression for the photovoltaic current can be rewritten in terms of the Green functions $\hat{G}^{R, A}\left(t, t^{\prime}\right)$ :

$I_{\mathrm{ph}}=2 e i \pi \nu \int_{0}^{\tau_{\mathrm{o}}} \frac{d t}{\tau_{\mathrm{o}}} \iint d t_{1} d t_{2} F_{i}^{\mathrm{ph}}\left(t_{1}, t_{2}\right) \sum_{i} \operatorname{tr}\left\{\hat{W}^{\dagger} \hat{G}^{R}\left(t, t_{1}\right) \hat{V}_{i} \hat{G}^{A}\left(t_{2}, t\right) \hat{W} \hat{\Lambda}\right\}$, 
which is more convenient in some calculations. Here the function

$F_{i}^{\mathrm{ph}}\left(t_{1}-t_{2}\right)=\left[\varphi_{i}\left(t_{1}\right)-\varphi_{i}\left(t_{2}\right)\right] f\left(t_{1}-t_{2}\right)$,

takes into account the probability of electron transitions due to the perturbation $\hat{V} \varphi_{i}(t)$ for the equilibrium electron distribution in the $\operatorname{dot} f(t)$. We notice, that taking higher order terms in $\hat{V}_{i}$ in $\hat{G}^{R, A}$ results in a new electron distribution function in the dot:

$\hat{V}_{i} F_{i}^{\mathrm{ph}}\left(t-t^{\prime}\right) \rightarrow \hat{G}^{R}\left(t, t_{1}\right) \hat{V}_{i} F_{i}^{\mathrm{ph}}\left(t_{1}-t_{2}\right) \hat{G}^{A}\left(t_{2}, t^{\prime}\right)$.

The effective electron distribution function has a shape different from the Fermi distribution function, see Sec. 5 .

We note that due to $\operatorname{tr} \hat{\Lambda}=0$ the expression for the conductance cannot be represented in terms of modified distribution function. As a result, see [46], conductance fluctuations are characterized by the electron temperature in the reservoirs rather than by the electron temperature in the dot. This statement was further investigated in [51, where the effect of time-dependent perturbation on two possible definitions of the conductance was studied. It was shown, that the Landauer conductance, defined as the linear response to the bias between the reservoirs and given by Eq. (15), is indeed characterized by the electron distribution function in the leads. In other geometries one can measure the linear response of electric current to the internal perturbation of the mesoscopic system by the dc electric field. Such response, called the Kubo conductance, is sensitive to the actual distribution function of electrons in the mesoscopic system.

\subsection{Current Noise}

The current correlation function $S$ represents fluctuations of the charge $Q=\int_{0}^{\tau_{\circ}} I(t) d t$ transported through the dot over the observation time interval $\tau_{\mathrm{o}}$

$S=\frac{\left\langle Q^{2}\right\rangle-\langle Q\rangle^{2}}{\tau_{\mathrm{o}}}=\int_{0}^{\tau_{\mathrm{o}}}\left(\left\langle I(t) I\left(t^{\prime}\right)\right\rangle-\langle I(t)\rangle\left\langle I\left(t^{\prime}\right)\right\rangle\right) \frac{d t d t^{\prime}}{\tau_{\mathrm{o}}}$.

Expression for $S$ in terms of the scattering matrices $\hat{\mathcal{S}}$ can be derived in a similar way to the derivation of Eq. (9) for current, and is outlined in Appendix B For arbitrary distribution function $f_{\alpha \beta}(t)=\delta_{\alpha \beta} f_{\alpha}(t)$ in the leads the current correlation function $S$ has the form $\left(\delta_{t, t^{\prime}}=\delta\left(t-t^{\prime}\right)\right)$ :

$$
\begin{aligned}
S & =\int_{0}^{\tau_{\mathrm{o}}} d t d t^{\prime} \int d t_{1} d t_{2} d t_{1}^{\prime} d t_{2}^{\prime} \operatorname{tr}\left\{\left(\hat{\mathcal{S}}^{\dagger}\left(t_{2}, t\right) \hat{\Lambda} \hat{\mathcal{S}}\left(t, t_{1}^{\prime}\right)-\hat{\Lambda} \delta_{t_{2}, t} \delta_{t, t_{1}^{\prime}}\right) \hat{f}\left(t_{1}^{\prime}-t_{2}^{\prime}\right)\right. \\
& \left.\times\left(\hat{\mathcal{S}}^{\dagger}\left(t_{2}^{\prime}, t^{\prime}\right) \hat{\Lambda} \hat{\mathcal{S}}\left(t^{\prime}, t_{1}\right)-\hat{\Lambda} \delta_{t_{2}^{\prime}, t^{\prime}} \delta_{t^{\prime}, t_{1}}\right)\left(\hat{1} \delta_{t_{1}, t_{2}}-\hat{f}\left(t_{1}-t_{2}\right)\right)\right\} .
\end{aligned}
$$

Below we consider the case of zero bias across the dot, so that $f_{\alpha}(t) \equiv f(t)$, see Eq. (10). We also assume that the temperature of the system $T$ is finite and $T \tau_{\mathrm{o}} \gg 1$. (The limit $T=0$ has some interesting properties and was discussed in Refs. [72 171). Then, $S$ can be divided into two parts:

$S=S_{\mathrm{NJ}}+S_{\mathrm{P}}$

Here, the second term $S_{\mathrm{P}}$ is chosen in such a way, that in the absence of time dependent perturbations this term vanishes $\left[S_{\mathrm{P}}=0\right.$, see Eq. (32) below], and only the first term remains. The first term describes the current noise due to thermal fluctuations of electrons in the leads at temperature $T$ and is known as the Nyquist-Johnson noise 90, 91]. 
The Nyquist-Johnson component of the noise can be written as

$S_{\mathrm{NJ}}=2 g_{\mathrm{cl}} T-\int_{0}^{\tau_{\mathrm{o}}} \frac{d t d t^{\prime}}{\tau_{\mathrm{o}}} \int d t_{1} d t_{2} f\left(t_{1}-t^{\prime}\right) \tilde{f}\left(t^{\prime}-t_{2}\right) \operatorname{tr}\left\{\hat{\Lambda} \hat{\mathcal{S}}\left(t, t_{1}\right) \hat{\Lambda} \hat{\mathcal{S}}^{\dagger}\left(t_{2}, t\right)\right\}$,

where $\tilde{f}(t)=\delta(t)-f(t)$. The first term in Eq. (31) represents the noise of a classical resistor with resistance $1 / g_{\mathrm{cl}}$. The second term in Eq. (31) describes the contribution to the current noise from the quantum mechanical corrections to the conductivity, cf. Eq. (15). In the absence of time-dependent perturbations, the second term represents the quantum correction to the conductance, so that the noise correlator has the form $S_{\mathrm{NJ}}=2 g T$, where $g$ is the sample-specific conductance of the dot, see Eq. (15).

The external field changes the conductance of the dot, see Sec. प Consequently, we can expect that the Nyquist-Johnson contribution to the current noise is also modified due to the external field. In particular, the ensemble average $S_{\mathrm{NJ}}$ and fluctuations of $S_{\mathrm{NJ}}$ with respect to different dot realizations are suppressed by timedependent perturbation.

The second term, $S_{\mathrm{P}}$, in Eq. (30) represents the noise of the photovoltaic current Eq. (18) and has the following form in terms of the scattering matrix $\hat{\mathcal{S}}\left(t, t^{\prime}\right)$ :

$$
\begin{aligned}
S_{\mathrm{P}} & =e^{2} \int_{0}^{\tau_{\mathrm{o}}} \frac{d t d t^{\prime}}{\tau_{\mathrm{o}}} \int d t_{1} d t_{2} d t_{1}^{\prime} d t_{2}^{\prime} f\left(t_{1}-t_{2}\right) \tilde{f}\left(t_{1}^{\prime}-t_{2}^{\prime}\right) \\
& \times \operatorname{tr}\left\{\hat{\mathcal{S}}\left(t_{2}^{\prime}, t\right) \hat{\Lambda} \hat{\mathcal{S}}^{\dagger}\left(t, t_{1}\right) \hat{\mathcal{S}}\left(t_{2}, t^{\prime}\right) \hat{\Lambda} \hat{\mathcal{S}}^{\dagger}\left(t^{\prime}, t_{1}^{\prime}\right)-\Lambda^{2} \delta_{t_{2}^{\prime}, t} \delta_{t, t_{1}} \delta_{t_{2}, t^{\prime}} \delta_{t^{\prime}, t_{1}^{\prime}}\right\} .
\end{aligned}
$$

To discuss the noise of the photovoltaic current in more detail, we consider the adiabatic limit, when the eigenvalues of the time-delay matrix Eq. (19) are shorter than both $1 / T$ and $1 / \omega_{i},\left(\omega_{i}\right.$ is the frequency of external perturbation $\left.\hat{V}_{i}\right)$. The ensemble average value of $S_{\mathrm{P}}$ for arbitrary strength and frequency of the perturbations was

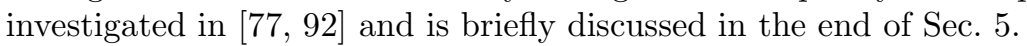

In the adiabatic limit only electrons close to the Fermi energy contribute to the current. Thus, we can neglect energy dependence of the scattering matrix $\hat{\mathcal{S}}(\varepsilon, t)$ in the Wigner representation Eq. (17) and substitute $\hat{\mathcal{S}}\left(t, t^{\prime}\right)=\hat{\mathcal{S}}_{t}(\varepsilon=0) \delta_{t, t^{\prime}}$ into Eq. (32):

$S_{\mathrm{P}}=e^{2} \int_{0}^{\tau_{\mathrm{o}}} \frac{d t d t^{\prime}}{\tau_{\mathrm{o}}} \operatorname{tr}\left\{\hat{\Lambda}^{2}-\hat{\mathcal{S}}_{t}^{\dagger}(0) \hat{\Lambda} \hat{\mathcal{S}}_{t}(0) \hat{\mathcal{S}}_{t^{\prime}}^{\dagger}(0) \hat{\Lambda} \hat{\mathcal{S}}_{t^{\prime}}(0)\right\} f\left(t-t^{\prime}\right) \tilde{f}\left(t-t^{\prime}\right)$.

We observe that the temporal correlations in the current survive on time scales comparable with the observation time $\tau_{\mathrm{o}}$ at low temperatures $T \tau_{\mathrm{o}} \ll 1$. In this case the full counting statistics is non-trivial and higher moments of the current should be investigated, see Refs. 72 71] for more detail. At finite temperature $T$, the temporal correlations of the current are suppressed on time scale of the order of $1 / T$, see Eq. (10), and the counting statistics of the current becomes Gaussian and is described by the average value of the current $I_{\mathrm{ph}}$, Eq. (18) and its noise $S_{\mathrm{P}}$, Eq. (32).

Within a bilinear response to the perturbation Eq. 23] we obtain the following expression for the noise

$S_{\mathrm{P}}=e^{2} F_{\mathrm{n}}(T, \omega)\left(\mathcal{K}_{11} X_{1}^{2}+\mathcal{K}_{22} X_{2}^{2}+2 \cos \phi \mathcal{K}_{12} X_{1} X_{2}\right)$.

Here coefficients $\mathcal{K}_{i j}$ are given by

$\mathcal{K}_{i j}=\operatorname{tr}\left\{\left[\hat{\Lambda} ; \hat{\mathcal{R}}_{i}\right]\left[\hat{\mathcal{R}}_{j} ; \hat{\Lambda}\right]\right\}$ 
and the function $F_{\mathrm{n}}(T, \omega)$ represents the probability of the absorption or emission of a perturbation quantum with energy $\omega$ :

$$
\begin{aligned}
F_{\mathrm{n}} & =\int \frac{d \varepsilon}{4 \pi}\left[f\left(\varepsilon+\frac{1}{2} \omega\right) \tilde{f}\left(\varepsilon-\frac{1}{2} \omega\right)-2 f(\varepsilon) \tilde{f}(\varepsilon)+f\left(\varepsilon-\frac{1}{2} \hbar \omega\right) \tilde{f}\left(\varepsilon+\frac{1}{2} \hbar \omega\right)\right] \\
& =\frac{\omega}{2 \pi}\left(\operatorname{coth} \frac{\omega}{2 T}-\frac{2 T}{\omega}\right) .
\end{aligned}
$$

At low temperatures $T \ll \omega$, but still $T \gg 1 / \tau_{\mathrm{o}}, F_{\mathrm{n}}=\omega / 2 \pi$. As $T$ increases, $F_{\mathrm{n}}$ decreases $F_{\mathrm{n}}=\omega^{2} / T$.

Above we discussed the current noise in the situation when the bias across the dot is zero. When a finite bias is applied, the noise acquires dependent on the bias contribution called shot noise. It was shown [93] that the shot noise originate only due to quantum corrections to electron transport, while the classical contribution to the transport does not lead to the shot noise. Therefore, one can expect that a time-dependent perturbation suppresses shot noise along with any other quantum interference characteristics of electron transport. Another interesting effect of microwave radiation on the shot noise of open quantum dots was found by Lamacraft in Ref. 94]. This effect results in cusps of the noise power when the bias $\mathrm{eV}$ is a multiple of microwave frequency $\omega: \mathrm{eV}=n \omega$ with integer $n$.

\section{Ensemble of Open Quantum Dots}

The exact form of the Hamiltonian Eq. (5) for quantum dots depends on many microscopic parameters of the system, such as the shape of the dot, position of impurities and is usually too complicated for analysis. However, for many purposes the interesting question is what the statistical properties of transport coefficients through a quantum dot, rather than the corresponding values for each particular sample. To describe statistical properties of quantum dots, a random matrix theory turns out to be a productive tool. The random matrix description of quantum dots is based on the assumption, that the Hamiltonian of the dot, Eq. (5), is determined by $M \times M$ matrices $\hat{H}$ and $\hat{V}_{i}$ with $\hat{H}$ being a random realization of a hermitian matrix from the Gaussian ensemble [29]. The matrix elements $H_{n m}(\Phi)$ of matrices from this ensemble in the presence of magnetic flux $\Phi$ through the dot are described by the following correlators

$\overline{H_{n m}\left(\Phi_{1}\right) H_{n^{\prime} m^{\prime}}^{*}\left(\Phi_{2}\right)}=\frac{M \delta_{1}^{2}}{\pi^{2}}\left[L\left(\Phi_{1}-\Phi_{2}\right) \delta_{n n^{\prime}} \delta_{m m^{\prime}}+L\left(\Phi_{1}+\Phi_{2}\right) \delta_{m n^{\prime}} \delta_{n m^{\prime}}\right]$,

Here $\overline{(\ldots)}$ stands for the ensemble averaging, and $\delta_{1}$ is the mean level spacing of eigenvalues of $\hat{H}$. For small $\Delta \Phi$ function $L(\Delta \Phi)$ can be estimated as $L(\Delta \Phi)=$ $1-\kappa\left(\Delta \Phi / \Phi_{\mathrm{q}}\right)^{2}$, where $\kappa$ is a non-universal, sample-specific constant of the order of unity, and $\Phi_{\mathrm{q}}=c / e$ is the flux quantum 95, 7]. At $\Phi_{1,2}=0$, the matrix $\hat{H}(0)$ belongs to the orthogonal ensemble. As $\Delta \Phi$ increases, $L(\Delta \Phi)$ vanishes, and $\hat{H}(\Phi)$ becomes a matrix from the unitary ensemble when the second tern in Eq. (37) is equal to zero. The microscopic justification of the random matrix description Eq. (37) can be found in [34, 35, 36] for disordered systems and in 37, 38, 12] in ballistic chaotic systems.

Matrices $\hat{V}_{i}$ can also considered as Hermitian random matrices. Below we disregard the fluctuations of the matrices $\hat{V}_{i}$, and assume that $\hat{V}_{i}$ are real symmetric 
matrices, belonging to an orthogonal ensemble. In this case, we characterize perturbations $\hat{V}_{i}$ by parameters

$C_{i j}=\frac{\pi}{M^{2} \delta_{1}} \operatorname{tr} \hat{V}_{i} \hat{V}_{j}$

We remind that $\operatorname{tr} \hat{V}_{i}=0$, see Eq. (7). The parameters $C_{i j}$ have the meaning of the level velocities which characterizing the evolution of an energy level $\varepsilon_{n}(\varphi)$ under the external perturbation $\sum_{i} \hat{V}_{i} \varphi_{i}(t)$, , 96, 11]:

$\frac{2 \delta_{1}}{\pi} C_{i j}=\overline{\frac{\partial \varepsilon_{\nu}}{\partial \varphi_{i}} \frac{\partial \varepsilon_{\nu}}{\partial \varphi_{j}}}-\overline{\frac{\partial \varepsilon_{\nu}}{\partial \varphi_{i}}} \frac{\overline{\partial \varepsilon_{\nu}}}{\partial \varphi_{j}}$.

Parameters $C_{i j}$ are also related to the transition rates of electrons under perturbation $\hat{V}_{i}$. Indeed, the transition rate $\gamma_{11}$ due to perturbation $\hat{V}_{1}$ is determined by the Fermi golden rule:

$\gamma_{11}=\sum_{m} 2 \pi\left|V_{1 ; n m}\right|^{2} \delta\left(\varepsilon_{n}-\varepsilon_{m} \pm \omega\right) \sim \frac{\overline{\left|V_{1 ; n m}\right|^{2}}}{\delta_{1}} \simeq \frac{C_{11}}{\pi}$.

The first equality sign follows from the Fermi golden rule, the second sign represents an estimate of the characteristic value of the matrix elements $\overline{\left|V_{1 ; n m}\right|^{2}}$ and the density of states $1 / \delta_{1}$, the last equation is the definition of $C_{11}$, cf. Eq. (38). If perturbations induce uniform electric fields $E_{i}$ in a quantum dot with typical length $L$, parameters $C_{i j}$ can be estimated as $C_{i j} \simeq e^{2} E_{i} E_{j} L^{2} / E_{\mathrm{Th}}$, where $E_{\mathrm{Th}} \sim M \delta_{1}$ is the Thouless energy.

Below we show that all statistical transport characteristics of quantum dots in the presence of time-dependent perturbations are functions of parameters $C_{i j}$. Thus, even though $C_{i j}$ are free parameters, measurements of several transport characteristics [10, 82, 83, allow one to eliminate the uncertainty of $C_{i j}$.

For calculations of different correlation functions of transport parameters over the ensemble of random Hamiltonians $\hat{H}$, we use a diagrammatic technique, similar to one developed for disordered metals, see [24]. In this Section, we briefly discuss the basic elements of this diagrammatic technique.

First, we calculate the ensemble averaged Green function $\hat{G}^{R, A}(\varepsilon)$ in the absence of time-dependent perturbations. The diagram equation in Fig. 2 reduces to the following algebraic equation for the electron self-energy $\Sigma(\varepsilon)=\left(M \delta_{1}^{2} / \pi^{2}\right) \operatorname{tr} \hat{G}^{R}(\varepsilon)$ :

$\Sigma(\varepsilon)=\frac{M \delta_{1}^{2}}{\pi^{2}} \frac{1}{\varepsilon-\Sigma(\varepsilon)+i 0}-N_{\mathrm{ch}} \frac{M \delta_{1}^{3}}{\pi^{2}} \frac{1}{\varepsilon-\Sigma(\varepsilon)+i 0} \frac{1}{\varepsilon-\Sigma(\varepsilon)+i M \delta_{1} / \pi}$.

Solving Eq. (41), we find the ensemble average Green function $\overline{\hat{G}^{R}(\varepsilon)}=\left(\overline{\hat{G}^{A}(\varepsilon)}\right)^{*}$ for $\varepsilon \ll M \delta_{1}$ in the form

$\overline{G_{n m}^{R}(\varepsilon)}=-i \delta_{m n} \frac{\pi}{M \delta_{1}} \begin{cases}1+\frac{N_{\mathrm{ch}}+i 2 \pi \varepsilon / \delta_{1}}{4 M}, & N_{\mathrm{ch}}<n \leq M ; \\ \frac{1}{2}, & 1 \leq n \leq N_{\mathrm{ch}} .\end{cases}$

In derivation of Eqs. (41) and (42), we used the following values for factors $\mathcal{T}$ in Eq. (2)

$\mathcal{T}=\sqrt{\frac{M \delta_{1}}{\pi^{2} \nu}}$

This choice of $\mathcal{T}$ corresponds to a dot connected to the leads by reflectionless contacts, when the ensemble averaged scattering matrix $\overline{\mathcal{S}_{\alpha \beta}}$ is zero and $\hat{\mathcal{S}}$ belongs to circular ensemble, see [7]. 


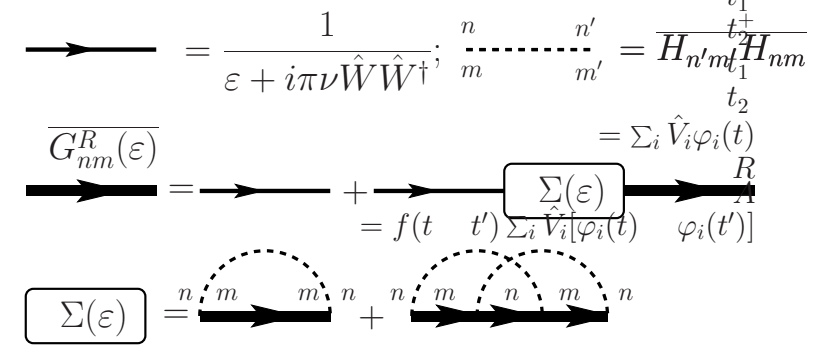

Figure 2. Diagrams for the ensemble averaged electron Green function in the dot. The first line of the figure introduces the bare Green function $[\varepsilon+$ $\left.i \pi \nu \hat{W} \hat{W}^{\dagger}\right]^{-1}$ and correlation function of the matrix elements of the Hamiltonian $\hat{H}$. The second line represents the Dyson-type equation for the ensemble averaged Green function $\overline{G_{n m}^{R}(\varepsilon)}$. The third line introduces the first two terms of the selfenergy $\Sigma(\varepsilon)$, which is diagonal in index of electron states in the dot. The second term as well as all other terms which contain intersections of dashed lines are small in parameter $1 / M$.

We also introduce two other elements of the diagram technique used in calculations of statistical properties of electron transport in the presence of timedependent perturbations. One element is called the diffuson $\mathcal{D}\left(t_{1}, t_{2}, \tau\right)$ and is defined by

$$
\begin{aligned}
\overline{\left[G_{n m ; \Phi_{1}}^{R}\left(t_{1}^{+}, t_{2}^{+}\right) G_{m n ; \Phi_{2}}^{A}\left(t_{2}^{-}, t_{1}^{-}\right)\right]_{\mathrm{amp}}=} & \frac{4 M^{2} \delta_{1}^{2}}{\pi^{2}} \delta\left(t_{1}^{+}++t_{2}^{-}-t_{2}^{+}-t_{1}^{-}\right) \\
& \times \mathcal{D}_{\Phi_{1}-\Phi_{2}}\left(\frac{t_{1}^{+}+t_{1}^{-}}{2}, \frac{t_{2}^{+}+t_{2}^{-}}{2}, t_{1}^{+}-t_{2}^{+}\right),
\end{aligned}
$$

see Fig. 3 a). The other element is called the Cooperon $\mathcal{C}\left(\tau_{1}, \tau_{2}, t\right)$ and is defined as

$$
\begin{aligned}
\overline{\left[G_{n m ; \Phi_{1}}^{R}\left(t_{1}^{+}, t_{2}^{+}\right) G_{n m ; \Phi_{2}}^{A}\left(t_{1}^{-}, t_{2}^{-}\right)\right]_{\mathrm{amp}}=} & \frac{4 M^{2} \delta_{1}^{2}}{\pi^{2}} \delta\left(t_{1}^{+}++t_{2}^{-}-t_{2}^{+}-t_{1}^{-}\right) \\
& \times \mathcal{C}_{\Phi_{1}+\Phi_{2}}\left(t_{1}^{+}-t_{1}^{-}, t_{2}^{+}-t_{2}^{-}, \frac{t_{1}^{+}+t_{1}^{-}}{2}\right),
\end{aligned}
$$

see Fig. [ b). These two elements represent the ensemble average product of the advanced and retarded electron Green functions in the dot, divided by the product $\overline{G_{n n ; \Phi_{1}}^{R}} \overline{G_{m m ; \Phi_{1}}^{R}} \overline{G_{n n ; \Phi_{2}}^{A}} \overline{G_{m m ; \Phi_{2}}^{A}}$, so-called the amputated average. The diffuson and the Cooperon are given by the following expressions:

$$
\begin{aligned}
& \mathcal{D}_{\Delta \Phi}\left(t_{1}, t_{2}, \tau\right)=\theta\left(t_{1}-t_{2}\right) \exp \left(-\int_{t_{2}}^{t_{1}} \Gamma_{\Delta \Phi}(\tau, t) d t\right) ; \\
& \mathcal{C}_{\Delta \Phi}\left(\tau_{1}, \tau_{2}, t\right)=\theta\left(\tau_{1}-\tau_{2}\right) \exp \left(-\frac{1}{2} \int_{\tau_{2}}^{\tau_{1}} \Gamma_{\Delta \Phi}(\tau, t) d \tau\right) .
\end{aligned}
$$

Here we use the notation

$$
\begin{aligned}
& \Gamma_{\Delta \Phi}(\tau, t)=\gamma_{\mathrm{esc}}+\gamma(\Delta \Phi)+\sum_{i j} \tilde{\varphi}_{i}(\tau, t) C_{i j} \tilde{\varphi}_{j}(\tau, t) \\
& \gamma_{\mathrm{esc}}=\frac{N_{\mathrm{ch}} \delta_{1}}{2 \pi} ; \quad \gamma(\Delta \Phi)=\frac{2 M \delta_{1}}{\pi}[1-L] ; \quad \tilde{\varphi}_{i}(\tau, t)=\varphi_{i}(t+\tau / 2)-\varphi_{i}(t-\tau / 2)
\end{aligned}
$$


a)

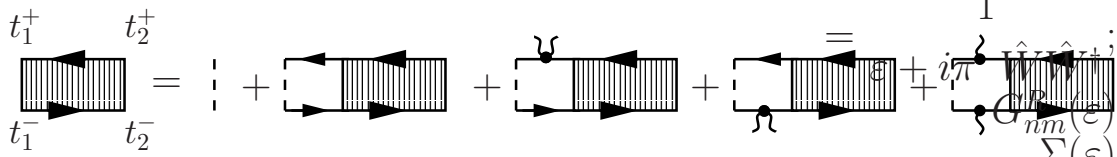

b)

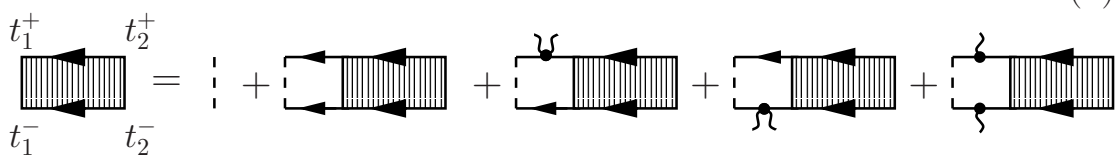

c) $\quad \boldsymbol{b}=\sum_{i} \hat{V}_{i} \varphi_{i}(t) \quad \mho s=?+3+$

Figure 3. The Dyson-type equations for the diffuson, Eq. 44), and the Cooperon, Eq. 45), are shown in the first and the second lines respectively. The subscript "amp" in Eqs. 44) and 45] emphasizes that the four Green functions at the terminals of the diffuson and the Cooperon are omitted. All Green functions in these diagrams and diagrams in figures below are ensemble average Green functions, introduced in the second line of Fig. 2

The first term in Eq. (48) is the electron escape rate from the dot and $\gamma_{\Delta \Phi}$ is the electron dephasing rate due to the difference in magnetic flux $\Delta \Phi$. The last term in Eq. (48) describes the effect of time-dependent field on the correlation functions Eqs. (44) and (45) of electron propagators. Equations (42), (46) and (47) are the building blocks of the diagrams, which are studied below for different correlation functions of transport characteristics of open quantum dots.

\section{Effect of time-dependent perturbations on the conductance}

\subsection{Weak localization correction}

Weak localization correction to the conductance of a quantum dot is given by the ensemble average of the second term in Eq. (15). For the unitary ensemble weak localization correction [97] is small as $g_{\mathrm{cl}} / N_{\mathrm{ch}}^{2} \ll 1$ and is beyond the accuracy of our calculations. In the orthogonal ensemble the weak localization correction is $g_{\mathrm{cl}} / N_{\mathrm{ch}} \sim 1$ [7]. We define the weak localization correction to the conductance as the difference between the averaged values of the conductance over orthogonal $(\Phi=0)$ and unitary $\left(\Phi \gg \Phi_{\mathrm{q}}\right)$ ensembles:

$\Delta g_{\mathrm{wl}}=\overline{(g)_{\Phi=0}}-\overline{(g)_{\Phi \gg \Phi_{\mathrm{q}}}}$.

In this subsection we describe the effect of time-dependent field on the weak localization correction Eq. (50).

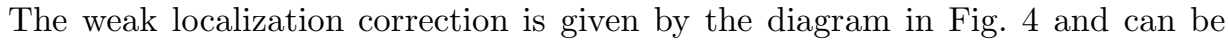
calculated from the following expression [45]:

$\Delta g_{\mathrm{wl}}=\Delta g_{\mathrm{wl}}^{(0)} \int_{0}^{2 \pi / \varnothing} \frac{\omega d t}{\pi} \int_{0}^{\infty} \gamma_{\mathrm{esc}} d \tau \mathcal{C}(\tau,-\tau, t), \quad \Delta g_{\mathrm{wl}}^{(0)}=-G_{0} \frac{N_{\mathrm{l}} N_{\mathrm{r}}}{N_{\mathrm{ch}}^{2}}$.

This equation gives the universal description of the effect of the time-dependent fields on the weak localization correction. Below we will discuss different asymptotic 


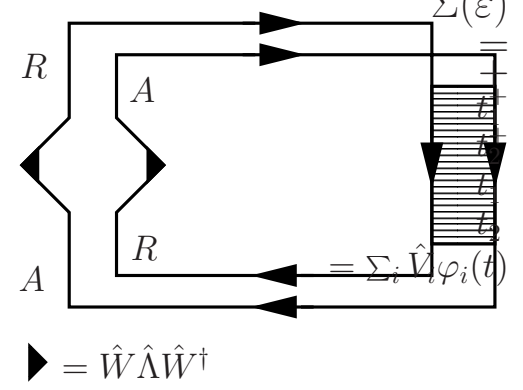

Figure 4. The diagram for the calculation of the weak localization correction to the conductance.

regimes for the case when the perturbation is described by only one harmonic function $\varphi_{1}(t)=\cos \omega t$ and $C_{11}=C_{1}$.

In the absence of the time dependent perturbation $C_{1} \equiv 0$, one obtains $\Delta g_{\mathrm{wl}}=$ $\Delta g_{\mathrm{wl}}^{(0)}$ [7, 98. For weak external field $C_{\mathrm{l}} \ll \gamma_{\text {esc }}$ we find

$\frac{\Delta g_{\mathrm{wl}}}{\Delta g_{\mathrm{wl}}^{(0)}}=1-\frac{\pi C_{\mathrm{l}}}{\gamma_{\mathrm{esc}}} \frac{\omega^{2}}{\omega^{2}+\gamma_{\mathrm{esc}}^{2}}$,

where $\gamma_{\text {esc }}$ is defined in Eq. (49). In this regime the correction is quadratic in the frequency of slowly oscillating field, similarly to the result for bulk metal system at $\omega$ smaller than the dephasing rate $1 / \tau_{\phi}$. However, the frequency dependence saturates at large frequency. It is different from the result for bulk systems [39, where a characteristic spatial scale shrinks as $\sqrt{D / \omega}$ with $D$ being the diffusion coefficient, whereas in a quantum dot this scale is determined by the size of the dot $L$. The random matrix description breaks down at $\omega \sim D / L^{2}=E_{\mathrm{Th}}, E_{\mathrm{Th}}$ is the Thouless energy.

In the opposite limit of strong external field $C_{1} \gg \gamma_{\text {esc }}$ we consider separately the cases of fast, $\omega \gg \gamma_{\text {esc }}$, and slow, $\omega \ll \gamma_{\text {esc }}$ oscillations. In the first case we have

$\frac{\Delta g_{\mathrm{wl}}}{\Delta g_{\mathrm{wl}}^{(0)}}=\sqrt{\frac{\gamma_{\mathrm{esc}}}{\pi C_{\mathrm{l}}}}$.

The $1 / \sqrt{C_{1}}$ power dependence of the quantum correction is similar to that for the bulk system. Contrary to the bulk systems, the result does not depend on the frequency $\omega$ for the reason mentioned above. In the case of slow field $\omega \ll \gamma_{\text {esc }}$, but still $C_{1} \omega^{2} \gg \gamma_{\text {esc }}^{3}$ (strong field) the weak localization correction to the conductance is

$\frac{\Delta g_{\mathrm{wl}}}{\Delta g_{\mathrm{wl}}^{(0)}}=\frac{\Gamma(1 / 6)}{\pi \Gamma(5 / 6)} \sqrt[3]{\frac{\pi \gamma_{\mathrm{esc}}^{3}}{9 C_{\mathrm{l}} \omega^{2}}}$

The power and frequency dependence of $\Delta g_{\mathrm{wl}}$ is again different from that in bulk disordered metals, $\Gamma(x)$ is the $\Gamma$-function.

\subsection{Conductance fluctuations}

Next we consider the fluctuations of the conductance $g$ over ensemble of random Hamiltonians $\hat{H}$. We notice that the fluctuations in $g$ originate only from the second term in Eq. (15), since in the model of fully chaotic quantum dots with open channels 
the classical conductance $g_{\mathrm{cl}}$ does not fluctuate. We have for $\delta g=g-g_{\mathrm{cl}}$ the following correlation function, which can be derived [46] from the diagrams shown in Fig. 5 ]

$\overline{\delta g_{\Phi_{1}} \delta g_{\Phi_{2}}}=\frac{g_{\mathrm{cl}}^{2}}{N_{\mathrm{ch}}^{2}} \gamma_{\mathrm{esc}}^{2} \int_{0}^{2 \pi / \omega} \frac{\omega^{2} d t d t^{\prime}}{4 \pi^{2}} \int_{0}^{\infty} d \tau F^{2}(\tau) \int_{\tau / 2}^{\infty} d \theta\left[K^{+}\left(t, t^{\prime}, \tau, \theta\right)+K^{-}\left(t, t^{\prime}, \tau, \theta\right)\right]$,

where the functions $K^{ \pm}\left(t, t^{\prime}, \tau, \theta\right)$ are given by

$$
\begin{aligned}
K^{+}\left(t, t^{\prime}, \tau, \theta\right)= & \mathcal{D}\left(\frac{t+t^{\prime}}{2}, \frac{t+t^{\prime}+\tau}{2}-\theta, t^{\prime}-t\right) \mathcal{D}\left(\frac{t+t^{\prime}}{2}, \frac{t+t^{\prime}-\tau}{2}-\theta, t-t^{\prime}\right), \\
K^{-}\left(t, t^{\prime}, \tau, \theta\right)= & \mathcal{C}\left(t-t^{\prime}+\theta-\frac{\tau}{2}, t-t^{\prime}-\theta+\frac{\tau}{2}, \frac{t+t^{\prime}-\theta}{2}+\frac{\tau}{4}\right) \\
& \times \mathcal{C}\left(t^{\prime}-t+\theta+\frac{\tau}{2}, t^{\prime}-t-\theta-\frac{\tau}{2}, \frac{t+t^{\prime}-\theta}{2}-\frac{\tau}{4}\right) .
\end{aligned}
$$

The two terms in Eq. (55) have different properties with respect to the magnetic flux $\Phi$ through the dot. Although at $\Phi=0$ both terms survive, at finite magnetic field, $\left|\Phi_{1,2}\right| \sim \Phi_{\mathrm{q}}$, only one of them remains: for $\Phi_{2} \approx \Phi_{1}$ the second term vanishes, and for $\Phi_{2} \approx-\Phi_{1}$ the first term vanishes. The values of the conductance correlation function at $\Phi_{2}= \pm \Phi_{1}$ characterize the symmetry of the conductance with respect to magnetic field inversion. If conductance is symmetric, $\overline{\delta g_{\Phi} \delta g_{\Phi}}=\overline{\delta g_{\Phi} \delta g_{-\Phi}}$. This equation is indeed valid in the absence of time-dependent perturbations [99, 22. As was shown in Refs. 46. 50, time-dependent perturbations may suppress the symmetry of the conductance with respect to magnetic field inversion, see e.g. Eq. (61) below.

In the presence of a single harmonic perturbation at frequency $\omega$ and with strength $C_{11}=C_{1}$, we can write the conductance correlation function in the form

$\overline{\delta g_{\Phi_{1}} \delta g_{\Phi_{2}}}=\frac{g_{\mathrm{cl}}^{2}}{N_{\mathrm{ch}}^{2}}\left[\frac{\gamma_{\mathrm{esc}}^{2}}{\gamma_{-}^{2}} Q^{+}\left(\frac{C_{\mathrm{l}}}{\gamma_{-}}, \frac{T}{\gamma_{-}}, \frac{\omega}{\gamma_{-}}\right)+\frac{\gamma_{\mathrm{esc}}^{2}}{\gamma_{+}^{2}} Q^{-}\left(\frac{C_{\mathrm{l}}}{\gamma_{+}}, \frac{T}{\gamma_{+}}, \frac{\omega}{\gamma_{+}}\right)\right]$,

where we used a shorthand $\gamma_{ \pm}=\gamma_{\text {esc }}+\gamma\left(\Phi_{1} \pm \Phi_{2}\right)$ with $\gamma_{\text {esc }}$ and $\gamma(\Delta \Phi)$ defined in Eq. (49). At $T=0, \Phi_{1,2}=0$, and in the absence of time-dependent perturbations $Q^{ \pm}=1$. Below we discuss the properties of the functions $Q^{ \pm}$in various regimes.

In the limit of high temperature, $T \gg \gamma_{\mathrm{esc}}$, we obtain

$Q^{ \pm}(x, y, z) \approx \frac{\pi^{2}}{3 y} \frac{1}{\sqrt{1+2 x}}$

The equality between functions $Q^{ \pm}$means that the conductance is symmetric with respect to magnetic field inversion. However, in low temperature limit $T \ll \gamma_{ \pm}$, we obtain for strong perturbation $C_{1} \gg \gamma_{\text {esc }}$

$Q^{+}(x, 0, z) \approx \frac{1}{2 \sqrt{2 x}}, \quad Q^{-}(x, 0, z) \approx \frac{1}{2 x}$.

Equation (58) with $Q^{ \pm}$given by Eq. (60) shows an important signature of the effect of time-dependent perturbations on the conductance - the violation of the Onsager symmetry:

$\frac{\overline{\delta g_{\Phi} \delta g_{-\Phi}}}{\overline{\delta g_{\Phi} \delta g_{\Phi}}}=\sqrt{\frac{2 \gamma_{\mathrm{esc}}}{C_{\mathrm{l}}}}, \quad \gamma(2 \Phi) \gg \gamma_{\mathrm{esc}}$.

This breakdown of the Onsager relation is a simple manifestation of lifting of the time reversal symmetry in the system with time dependent Hamiltonian. 
In the limit of low frequency $\omega \ll \gamma_{\mathrm{esc}}$, the conductance $g$ can be represented as the result of averaging of the conductance $g\left(\left\{\varphi_{i}\right\}\right)$ at stationary perturbation $\varphi_{i}$ over one period $2 \pi / \omega$ :

$g=\int_{0}^{2 \pi / \omega} g\left(\left\{\varphi_{i}(t)\right\}\right) \frac{\omega d t}{2 \pi}$,

where $g\left(\left\{\varphi_{i}\right\}\right)$ can be calculated according to Eq. (15) with the scattering matrix defined by Eqs. (12) and (13) at fixed values $\varphi_{i}$. Because $g(\varphi)$ has magnetic field symmetry [22], $g$ is also symmetric with respect to inversion of magnetic field. Calculations of Eq. (55) at $\omega \ll \gamma_{\text {esc }}$ give

$Q^{ \pm}(x, y, 0)=\int_{0}^{2 \pi} \frac{d \xi d \zeta}{4 \pi^{2}} \int_{0}^{\infty} F^{2}\left(\xi / \gamma_{\mp}\right) \frac{\exp \left(-\left(1+4 x \sin ^{2} \xi / 2 \sin ^{2} \zeta / 2\right) \xi\right)}{1+4 x \sin ^{2} \xi / 2 \sin ^{2} \zeta / 2} d \xi$.

This expression in the limit of high temperature $T \gg \gamma_{\text {esc }}$ has the asymptote

$Q^{ \pm}(x, y, 0)=\frac{\pi}{3 y} K(-4 x)$,

and at zero temperature $Q^{ \pm}(x, 0,0)$ is given by

$Q^{ \pm}(x, 0,0)=\frac{1}{\pi} \frac{E(-4 x)+(1+4 x) K(-4 x)}{1+4 x}$,

where $K(x)$ and $E(x)$ are the elliptic integrals of the first and second kind respectively.

Suppression of the conductance fluctuations by slow field $\omega \ll \gamma_{\text {esc }}$, see Eq. (64) and (65) is the consequence of averaging of the stationary conductance $g(\varphi)$ over different configurations of the full Hamiltonian along the closed contour in parameter space, see Fig. 1 b). Thus, the observed d.c. conductance $g$, Eq. (62), is already partially averaged over ensemble of random Hamiltonians $\hat{H}$ and its fluctuations are reduced. As the strength of the perturbation $C_{1}$ increases, more statistically independent configurations of Hamiltonian $\hat{H}$ contribute to the conductance $g$ and fluctuations of $g$ become suppressed.

However, low-frequency perturbations do not affect the weak localization correction to the conductance $\Delta g_{\mathrm{wl}}$, Eq. (50), which is defined as the difference between the averages over orthogonal and unitary ensembles. Only perturbations at frequencies $\omega \sim \gamma_{\text {esc }}$ could suppress $\Delta g_{\mathrm{wl}}$, see e.g. Eq. (52), when the conductance $g$, Eq. (15), is no longer related to the stationary conductance $g(\varphi)$. In this case the suppression of both conductance fluctuations and the weak localization correction to the conductance are qualitatively similar and can be interpreted as dephasing.

\section{Photovoltaic current}

Photovoltaic current averaged over ensemble of random Hamiltonian $\hat{H}$ is zero, because there is no specific direction for the current to flow. However, for each particular configuration of the quantum dot, a finite current can flow in either direction. To characterize the value of this current, one can find [61, 47]

$\operatorname{var} I_{\mathrm{ph}}=\frac{\omega^{2} e^{2}}{4 \pi^{2}} \frac{N_{\mathrm{l}} N_{\mathrm{r}}}{N_{\mathrm{ch}}^{2}} \int_{0}^{2 \pi / \omega} \frac{\omega^{2} d t d t^{\prime}}{\pi^{2}} \int_{0}^{\infty} \gamma_{\mathrm{esc}}^{2} d \tau \int_{\tau / 2}^{\infty} d \theta K^{+}\left(t, t^{\prime}, \tau, \theta\right) B\left(t-\theta, t^{\prime}-\theta, \tau\right)$, 

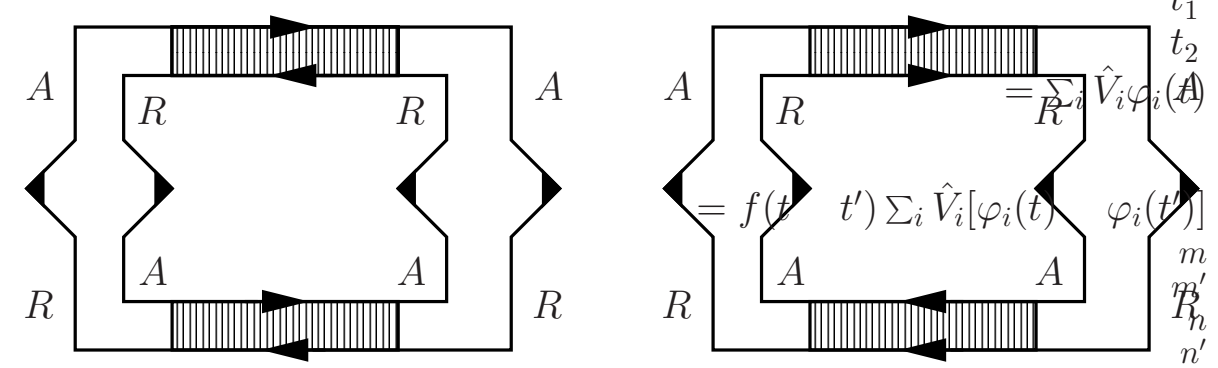

Figure 5. The diagrams for the calculation of the variance of conductance fluctuations.

where $K^{+}$is defined in Eq. (56) and

$$
\begin{aligned}
& B\left(t, t^{\prime}, \tau\right)=\gamma_{\mathrm{esc}}^{2} f^{2}(\tau) \int_{0}^{\infty} d \xi d \xi^{\prime} \mathcal{D}(t, t-\xi, \tau) \mathcal{D}\left(t^{\prime}, t^{\prime}-\xi^{\prime}, \tau\right)\left[\sum_{i j} \frac{C_{i j}}{\gamma_{\mathrm{esc}}} \tilde{\varphi}_{i}(\tau, t) \tilde{\varphi}_{j}\left(\tau, t^{\prime}\right)\right. \\
& \left.+2\left(\sum_{i j} \frac{C_{i j}}{\gamma_{\mathrm{esc}}} \tilde{\varphi}_{i}(\tau, t-\xi) \tilde{\varphi}_{j}(\tau, t-\xi)\right)\left(\sum_{i j} \frac{C_{i j}}{\gamma_{\mathrm{esc}}} \tilde{\varphi}_{i}\left(\tau, t^{\prime}-\xi^{\prime}\right) \tilde{\varphi}_{j}\left(\tau, t^{\prime}-\xi^{\prime}\right)\right)\right]
\end{aligned}
$$

with $\tilde{\varphi}_{i}(\tau, t)$ introduced in Eq. (49). In Fig. [6 we present only the diagram which survives at high temperatures and the full set of diagrams contributing to Eq. (66) can be found in Refs. 61, 47, We emphasize that the photovoltaic current has no symmetry with respect to inversion of magnetic field, $\overline{I_{\mathrm{ph}}(\Phi) I_{\mathrm{ph}}(-\Phi)}=0$, this statement in the diagrammatic language means that there is no counterpart of the diagram in Fig. 6] that contains the Cooperons; cf. Fig. [5

Function $B\left(t, t^{\prime}, \tau\right)$, Eq. (67), is related to the electron distribution function in the dot. Particularly, at high temperature $T \gg T_{\mathrm{h}}$ for harmonic perturbations at frequency $\omega$

$B\left(t, t^{\prime}, \tau\right)=\sum_{i j} C_{i j} \dot{\varphi}_{i}\left(\frac{t+t^{\prime}}{2}\right) \dot{\varphi}_{j}\left(\frac{t+t^{\prime}}{2}\right)\left(\frac{T \sin (\omega \tau / 2)}{\omega^{2} \sinh \pi T \tau}\right)^{2}$,

corresponds to a square of the distribution function $F_{i}^{\mathrm{ph}}$, Eq. (26). Here we introduced temperature scale $T_{\mathrm{h}}$ according to

$T_{\mathrm{h}}=\omega \max \left\{\sqrt{\frac{C_{i j}}{\gamma_{\mathrm{esc}}}}\right\}$.

For a single perturbation at high frequency $\omega \gg \gamma_{\text {esc }}$ with strength $C_{1}$ and at low temperature $T \ll T_{\mathrm{h}}$, we can estimate the integrals over $\xi$ and $\xi^{\prime}$ in Eq. (67) as

$\int_{0}^{\infty} \mathcal{D}(t, t-\xi, 2 \tau) d \xi \approx \frac{\gamma_{\mathrm{esc}}}{\gamma_{\mathrm{esc}}+2 C_{\mathrm{l}} \sin ^{2} \omega \tau}$,

and the function $B\left(t, t^{\prime}, \tau\right)$ acquires extra factors Eq. (70), which vanish at $\tau \gg 1 / T_{\mathrm{h}}$. Narrowing of the distribution functions in time representation means the broadening of electron distribution in the energy space. Indeed, energy of an electron in the dot changes due to the external field. Such changes result in the redistribution of the electrons in the energy space and the new distribution function becomes wider than that of electrons in the leads at temperature $T$. 


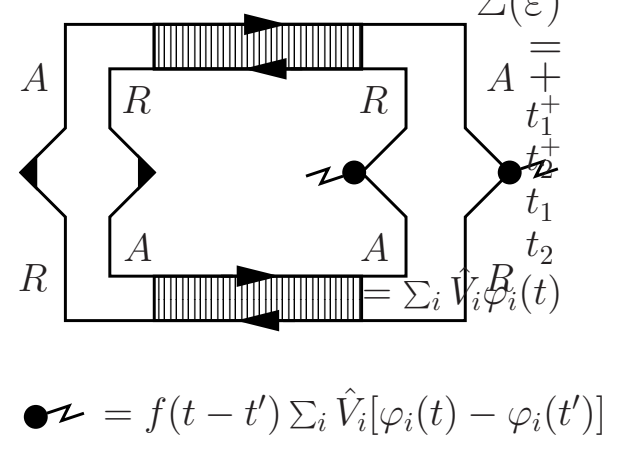

Figure 6. The diagram representing the contribution to the variance of the photovoltaic current, $\operatorname{var} I_{\mathrm{ph}}$, at high temperature $T$.

The new width of the electron distribution function can be estimated from the following argument. The transitions occur with rate $C_{\mathrm{l}}$, and electron stays in the dot for $1 / \gamma_{\text {esc }}$ time, so that it experiences of the order $C_{\mathrm{l}} / \gamma_{\text {esc }}$ transitions. After each transition electron energy changes by $\pm \omega$, and assuming that its motion in the energy space can be described by a random walk, we find that on average electron energy changes by $T_{\mathrm{h}}=\omega \sqrt{C_{\mathrm{l}} / \gamma_{\mathrm{esc}}}$, which is consistent with the estimate Eq. (70). The above estimate of $T_{\mathrm{h}}$ has a meaning only for strong fields, $C \gg \gamma_{\mathrm{esc}}$, so that the diffusion picture in energy space is valid. Otherwise, electrons experience only a few transitions with energy change $\omega$. Note that function in Eq. (70) is periodic in $\tau$ with period $\sim 1 / \omega$, i.e. at longer times the electron diffusion is no longer described by random walk and some structure in the distribution function appears [100].

For weak harmonic perturbation $C_{i j} \ll \gamma_{\text {esc }}$, we expand $K^{+}$to first order in $C_{i j}$ and neglect the second term in Eq. [67), since $T_{\mathrm{h}} \ll T$. As the result we obtain for the harmonic perturbation, characterized by two functions $\varphi_{1}(t)=\cos \omega t$ and $\varphi_{2}(t)=\cos (\omega t+\phi):$

$\operatorname{var} I_{\mathrm{ph}}=e^{2} \omega^{2} \frac{N_{\mathrm{l}} N_{\mathrm{r}}}{N_{\mathrm{ch}}^{2}} \int_{0}^{\infty} \gamma_{\mathrm{esc}} d \theta e^{-2 \gamma_{\mathrm{esc}} \theta} \int_{-\theta}^{+\theta} d \tau B(\tau) \frac{C_{\mathrm{l}}^{2}(2 \omega \theta-\sin 2 \omega \theta)+C_{\mathrm{c}}^{2} \sin 2 \omega \theta}{\omega}$,

where the linear, $C_{\mathrm{l}}$, and circular, $C_{\mathrm{c}}$, perturbation amplitudes were introduced according to

$C_{\mathrm{l}}=C_{11}+2 C_{12} \cos \phi+C_{22}, \quad C_{\mathrm{c}}=2 \sin \phi \sqrt{C_{11} C_{22}-C_{12}^{2}}$.

Note that the separation of the variance of the current into circular and linear contributions corresponds to the classification of the current components introduced in Ref. 65], where current through a microjunction in microwave field with linear and circular polarizations was studied. In the case of temperature $T$ larger than the escape rate, $T \gg \gamma_{\text {esc }}$, we have

$\operatorname{var} I_{\mathrm{ph}}=\frac{\pi}{12} \frac{e^{2} \omega^{2}}{4 \pi^{2}} \frac{N_{\mathrm{l}} N_{\mathrm{r}}}{N_{\mathrm{ch}}^{2}} \frac{\gamma_{\mathrm{esc}}}{T} \frac{1}{\gamma_{\mathrm{esc}}^{2}+\omega^{2}}\left(\frac{\omega^{2}}{\gamma_{\mathrm{esc}}^{2}} C_{\mathrm{l}}^{2}+C_{\mathrm{c}}^{2}\right)$.

The second term of Eq. (73) survives the limit $\omega \rightarrow 0$, thus reproducing the known result for adiabatic pumping [57, 59]. On the other hand, this term vanishes at high 
frequency. The $C_{1}^{2}$ term is quadratic in frequency at small frequency and tends to a constant at large frequency.

The linear pumping amplitude $C_{1}$ in the case of two pumps has the form of Eq.(72), which implies that the amplitude $C_{1}$ is just a vector sum of different pumps in the parameter space. On the other hand the circular amplitude is determined by "uncorrelated" components of matrices $\hat{V}_{1,2}$ and vanishes if $\hat{V}_{2} \propto \hat{V}_{1}$.

To describe the variance of the photovoltaic current, we first consider the adiabatic limit, when parameters $C_{i j}$ have a special form $C_{11}=C_{22}=C$ and $C_{12}=0$. In this case the expression for the variance can be written in the compact form for $T \gg \gamma_{\text {esc }}$

$\operatorname{var} I_{\mathrm{ph}}=\frac{e^{2} \omega^{2}}{24 \pi} \frac{N_{\mathrm{l}} N_{\mathrm{r}}}{N_{\mathrm{ch}}^{2}} \frac{\gamma_{\mathrm{esc}}}{T} \frac{2 C+\left(\gamma_{\mathrm{esc}}-\sqrt{\gamma_{\mathrm{esc}}\left(\gamma_{\mathrm{esc}}+4 C\right)}\right)}{\sqrt{\gamma_{\mathrm{esc}}\left(\gamma_{\mathrm{esc}}+4 C\right)}}$.

As temperature drops down to $T \ll \gamma_{\mathrm{esc}}=N_{\mathrm{ch}} \delta_{1} / 2 \pi$, the variance of $I_{\mathrm{ph}}$ saturates to

$\operatorname{var} I_{\mathrm{ph}}=\frac{e^{2} \omega^{2}}{\pi^{2}} \frac{N_{\mathrm{l}} N_{\mathrm{r}}}{N_{\mathrm{ch}}^{2}} \frac{C^{2}}{\sqrt{\gamma_{\mathrm{esc}}\left(\gamma_{\mathrm{esc}}+4 C\right)^{3}}}$.

The authors of Ref. 59 showed that at strong perturbation the variance of the photovoltaic current in the adiabatic limit is proportional to the perimeter of the contour integral in the parameter space Fig. $1 \mathrm{~b}$ ); $\operatorname{var} I_{\mathrm{ph}} \propto \sqrt{C}$. This perimeter law is the consequence of the lack of correlation between distant points of the contour in the parameter space. The total contribution to the pumped current consists of uncorrelated contributions of the loop and is proportional to the number of independent contributions $\sqrt{C / \gamma_{\text {esc }}}$. In the opposite case of weak perturbation $C \ll \gamma_{\text {esc }}$ the current $I_{\text {ph }}$ is determined by Eq. (24), see [57] and is proportional to $C_{\mathrm{c}}$ $\left(\operatorname{var} I_{\mathrm{ph}} \propto C_{\mathrm{c}}^{2}\right)$. Equations (74) and (75) are consistent with the above arguments for power dependence of var $I_{\mathrm{ph}}$.

When only one perturbation $\varphi_{i}(t)$ with power $C_{1}$ is applied, the photovoltaic current vanishes in the adiabatic limit. In this case the photovoltaic current is quadratic in frequency $\omega$ for $\omega \ll \gamma_{\text {esc }}$. For weak pumping $\operatorname{var} I_{\mathrm{ph}}$ is determined by Eq. (73) with $C_{\mathrm{c}}=0$ for arbitrary frequency $\omega$. For strong pumping $C_{\mathrm{l}} \gg \gamma_{\mathrm{esc}}$, but still low frequency limit $\omega^{2} C_{l} \ll \gamma_{\text {esc }}^{3}$, we have

$\operatorname{var} I_{\mathrm{ph}}=\frac{25}{288} \frac{e^{2} \omega^{2}}{4 \pi^{2}} \frac{N_{\mathrm{l}} N_{\mathrm{r}}}{N_{\mathrm{ch}}^{2}} \frac{\omega^{2}}{\gamma_{\mathrm{esc}}^{2}} \frac{\gamma_{\mathrm{esc}}}{T}\left(\frac{C_{\mathrm{l}}}{\gamma_{\mathrm{esc}}}\right)^{3 / 2}$.

In the limit of high frequencies, $T \gg \omega \gg \gamma_{\text {esc }}$, the variance of the photovoltaic current is given by

$\operatorname{var} I_{\mathrm{ph}}=\frac{e^{2} \omega^{2}}{24 \pi} \frac{N_{\mathrm{l}} N_{\mathrm{r}}}{N_{\mathrm{ch}}^{2}} \frac{\gamma_{\mathrm{esc}}}{T} \frac{C_{\mathrm{l}}+\gamma_{\mathrm{esc}}-\sqrt{\gamma_{\mathrm{esc}}\left(\gamma_{\mathrm{esc}}+2 C_{1}\right)}}{\sqrt{\gamma_{\mathrm{esc}}\left(\gamma_{\mathrm{esc}}+2 C_{\mathrm{l}}\right)}}$.

In the limit of strong pumping this expression has the $\sqrt{C_{1}}$ asymptotic behavior.

The results for $\operatorname{var} I_{\mathrm{ph}}$ at finite frequency have the following interpretation. Based on Eq. (21), we can represent the photovoltaic current in the form similar to Eq. (22), where the contour of integration is considered in the phase space. The phase space includes both parameters $\varphi_{i}(t)$ and their time derivatives [61, as follows from Eq. (21). At weak perturbation with a single parameter $\varphi_{1}(t)=\cos \omega t$, the contour is an ellipse with semi-axes proportional to $\sqrt{C_{1}}$ and $\omega \sqrt{C_{1}}$; then $I_{\mathrm{ph}} \propto(e \omega) \omega C_{\mathrm{l}}$ and $\operatorname{var} I_{\mathrm{ph}}$ is consistent with Eq. (73) at $\omega \ll \gamma_{\text {esc }}$. In the limit of strong perturbation at low frequency $\omega^{2} C_{\mathrm{l}} \ll \gamma_{\mathrm{esc}}^{3}$, the contour in phase plane is long along the $\varphi_{1}$ axis but narrow in the $\dot{\varphi}_{1}$ direction. The variance of the photovoltaic current is determined by a sum 
of independent contributions from pairs of the contour along the $\varphi$ axis, the number of these pairs can be estimated as $\sqrt{C_{\mathrm{l}} / \gamma_{\mathrm{esc}}}$. Each pair consists of two adjacent pieces of the contour shifted with respect to each other along $\dot{\varphi}_{1}(t)$ axis and contributes as $\omega \sqrt{C_{1}}$ to the total current. As a result, we obtain $\operatorname{var} I_{\mathrm{ph}} \propto(e \omega)^{2} \omega^{2} C_{1}^{3 / 2}$. Finally, if the amplitude of the field $C$ or the frequency $\omega$ increases further, $\omega^{2} C_{1} \gg \gamma_{\text {esc }}^{3}$, the contour does not have adjacent parts and each part of the contour gives an independent contribution. Since the number of this parts is $\sqrt{C_{\mathrm{l}} / \gamma_{\mathrm{esc}}}$, the variance of the photovoltaic current is proportional to $(e \omega)^{2} \sqrt{C}$, see Eq. (777).

As frequency $\omega$ and power increase further, the heating effects become important. At $T_{\mathrm{h}} \gg T$, the variance of the photovoltaic current can be roughly estimated if electron temperature $T$ in the leads is replaced by $T_{\mathrm{h}}$. Particularly, from Eq. (77) we obtain the characteristic scale for $\operatorname{var} I_{\mathrm{ph}}$ :

$\operatorname{var} I_{\mathrm{ph}} \sim \frac{e^{2} \omega^{2}}{4 \pi^{2}} \frac{N_{\mathrm{l}} N_{\mathrm{r}}}{N_{\mathrm{ch}}^{2}} \frac{\gamma_{\mathrm{esc}}}{\omega}$.

A numerical analysis 83] shows that in fact at $C_{1} \gg \gamma_{\mathrm{esc}}$, the variance of the photovoltaic current on the perturbation power has a very weak (log-like) dependence on $C_{\mathrm{l}}$, with typical of value $\operatorname{var} I_{\mathrm{ph}}$ consistent with the estimate of Eq. (78).

The heating effects manifest themselves in the noise of the photovoltaic current as well [77. In the limit of strong perturbation $C_{1} \gg \gamma_{\text {esc }}$ at high frequency $\omega \gg \gamma_{\text {esc }}$ the ensemble averaged value of $S_{\mathrm{P}}$, Eq. (32), is

$\overline{S_{\mathrm{P}}} \propto g_{\mathrm{cl}} T_{\mathrm{h}}$.

The noise of the photovoltaic current has a form similar to the expression for the Nyquist-Johnson noise, see Eq. (31): the current noise correlation function is determined by the conductance of the dot $g_{\mathrm{cl}}$, and the effective electron temperature. Due to the heating by a strong perturbation, the electron distribution function is broadened and the new energy scale for the electron distribution function is given by $T_{\mathrm{h}}$, see Eq. (79). Thus, the noise of the photovoltaic current averaged over the ensemble has a similar origin with the Nyquist-Johnson noise and is determined by thermal fluctuations of electrons in the dot out of equilibrium.

\section{Conclusions}

In summary, we reviewed the random matrix description of electron transport through an open quantum dot, subject to time-dependent perturbations. We expressed the dc current through the dot in terms of the scattering matrices, and considered such components of the current as the photovoltaic current, independent from the bias voltage, and the linear in the bias current, characterized by the conductance. The scattering matrices are calculated in terms of time-dependent Hamiltonian, that belongs to a Gaussian ensemble of random matrices. We then presented the diagram technique to perform ensemble averaging and applied this technique to calculate different statistical properties of the electron transport through the dot.

The main results can be summarized as follows. The weak localization correction to the conductance and conductance fluctuations are both suppressed by time-dependent perturbation. However, the suppression has different parametric dependence on perturbation frequency. The photovoltaic current can be represented as a sum of circular and linear terms. These term have different frequency dependence: the circular term dominates at low frequencies and represents the adiabatic charge 
pumping, while the linear term dominates at high frequencies. The photovoltaic current and its noise are determined by the actual width of the electron distribution function in the dot, on the other hand, the variance of the conductance fluctuations is determined by electron temperature in the leads. These results are in qualitative agreement with experiments, described in Refs. [10, 82.

We described calculations using the Hamiltonian approach to the statistical description of the electron transport, a detailed description of the scattering matrix approach for time-dependent system can be found in Ref. [47, where the same results were obtained.

In this review we considered the electron system neglecting the interaction effects and assumed spin degeneracy. The effect of electron-electron interaction can be disregarded only in the limit of the large number of open channels, but as the number of open channels decreases, the interaction effects become more important [84, 86, 87, 101]. The interplay of the interaction and time-dependent perturbation was addressed in Refs. 102, 103].

In semiconductor quantum dots in absence of magnetic field electron spin states are nearly degenerate. However, if magnetic field is applied, the spin degeneracy is lifted and currents of electrons with opposite spin orientations are not identical. In this case a spin current can be generated by time-dependent perturbation, similar to the photovoltaic charge current [104, this effect was studied experimentally in Ref. [105]. Another modification of the system, considered in the present review, is a quantum dot connected to superconducting leads and was studied theoretically in [106, 107, the experimental realization of such a system remains a challenging task.

\section{Acknowledgements}

I would like to thank I. Aleiner, V. Ambegaokar, P. Brouwer, L. DiCarlo, C. Marcus and M. Polianski, with whom I had a pleasure of working on various projects related to the topic of this review. Discussions with B. Altshuler, M. Büttiker, A. Clerk, V. Falko, and V. Kravtsov and A. D. Stone are greatly appreciated. This work was supported by the W. M. Keck Foundation and by NSF Materials Theory grant DMR-0408638.

\section{Appendix A.}

We denote the wave function of electrons in channel $\alpha$ by $\psi_{\alpha}(x, t)$ with $x<0$ for incoming electrons and $x>0$ for outgoing electrons, see Fig. 1 a). The boundary $x=0$ is described by a superposition of the incoming and outgoing electron states and we denote it by $\psi_{\alpha}(0, t)$. The wave function of electrons in the dot is denoted by $\psi_{i}(t)$.

We introduce the matrix Green function

$\hat{\mathcal{G}}_{\alpha \beta}\left(t, t^{\prime}, x, x^{\prime}\right)=\left(\begin{array}{cc}\mathcal{G}_{\alpha \beta}^{(R)}\left(t, t^{\prime}, x, x^{\prime}\right) & \mathcal{G}_{\alpha \beta}^{(K)}\left(t, t^{\prime}, x, x^{\prime}\right) \\ 0 & \mathcal{G}_{\alpha \beta}^{(A)}\left(t, t^{\prime}, x, x^{\prime}\right)\end{array}\right)$,

which is defined in terms of the retarded, advanced and Keldysh components as

$\mathcal{G}_{\alpha \beta}^{(R)}\left(t, t^{\prime}, x, x^{\prime}\right)=-i \Theta\left(t-t^{\prime}\right)\left\langle\left\{\psi_{\alpha}(x, t) ; \psi_{\beta}^{\dagger}\left(x^{\prime}, t^{\prime}\right)\right\}\right\rangle$,

$\mathcal{G}_{\alpha \beta}^{(A)}\left(t, t^{\prime}, x, x^{\prime}\right)=i \Theta\left(t^{\prime}-t\right)\left\langle\left\{\psi_{\alpha}(x, t) ; \psi_{\beta}^{\dagger}\left(x^{\prime}, t^{\prime}\right)\right\}\right\rangle$,

$\mathcal{G}_{\alpha \beta}^{(K)}\left(t, t^{\prime}, x, x^{\prime}\right)=-i\left\langle\left[\psi_{\alpha}(x, t) ; \psi_{\beta}^{\dagger}\left(x^{\prime}, t^{\prime}\right)\right]\right\rangle$, 
where $[A ; B]=A B-B A$ and $\{A ; B\}=A B+B A$. The similar expressions can be written down for $\hat{\mathcal{G}}_{i \alpha}\left(t, t^{\prime}, x^{\prime}\right)$ Green function, with $\psi_{\alpha}(x, t)$ replaced by $\psi_{i}(t)$.

For non-interacting electrons, moving towards the $\operatorname{dot}\left(x ; x^{\prime}<0\right)$, the Green's function is:

$\mathcal{G}_{\alpha \beta}\left(t, t^{\prime}, x, x^{\prime}\right)=\left(\begin{array}{cc}G_{\alpha \beta}^{R}\left(t-t^{\prime}, x-x^{\prime}\right) & G_{\alpha \beta}^{K}\left(t-t^{\prime}, x-x^{\prime}\right) \\ 0 & G_{\alpha \beta}^{A}\left(t-t^{\prime}, x-x^{\prime}\right)\end{array}\right)$,

where

$G_{\alpha \beta}^{R}(t, x)=i \Theta(t) \delta_{\alpha \beta} \delta\left(v_{F} t-x\right)$,

$G_{\alpha \beta}^{A}(t, x)=-i \Theta(-t) \delta_{\alpha \beta} \delta\left(v_{F} t-x\right)$,

$G_{\alpha \beta}^{K}(\varepsilon, x)=\tilde{f}_{\alpha}(\varepsilon)\left(G_{\alpha \beta}^{R}(\varepsilon, x)-G_{\alpha \beta}^{A}(\varepsilon, x)\right)$,

and $f(\varepsilon)$ is the distribution function of electrons in channel $\alpha$. If incoming electrons are in equilibrium at temperature $T$,

$\tilde{f}_{\alpha}(\varepsilon)=\tanh \frac{\varepsilon-e V_{\alpha}}{2 T}$,

with $V_{\alpha}$ being the voltage applied to the reservoir connected to the dot by channel $\alpha$.

The equations of motion for the Green functions $\hat{\mathcal{G}}_{\alpha \beta}\left(t, t^{\prime}, x, x^{\prime}\right)$ and $\hat{\mathcal{G}}_{j \alpha}\left(t, t^{\prime}, x^{\prime}\right)$ are

$i\left[\frac{\partial}{\partial t}-v_{F} \frac{\partial}{\partial x}\right] \hat{\mathcal{G}}_{\alpha \beta}\left(t, t^{\prime}, x, x^{\prime}\right)=\delta(x) W_{\alpha i} \hat{\mathcal{G}}_{i \beta}\left(t, t^{\prime}, x^{\prime}\right)+\delta\left(t-t^{\prime}\right) \delta\left(x-x^{\prime}\right) \hat{1}$,

$$
\left[i \frac{\partial}{\partial t}-H_{i j}(t)\right] \hat{\mathcal{G}}_{j \alpha}\left(t, t^{\prime}, x^{\prime}\right)=W_{i \beta}^{\dagger} \hat{\mathcal{G}}_{\beta \alpha}\left(t, t^{\prime}, 0, x^{\prime}\right) .
$$

Due to causality principle, $G_{\alpha \beta}^{A}\left(t, t^{\prime}, 0, x^{\prime}\right) \equiv 0$ for $x^{\prime}<0$. This observation significantly simplifies further calculations. Indeed, we can represent the Keldysh component of the Green's function in the left hand side of Eq. A.8) in the form

$\mathcal{G}_{i \alpha}^{(K)}\left(t, t^{\prime}, x^{\prime}\right)=\int d t_{1}\left[\frac{1}{i \partial / \partial t-\hat{H}(t)}\right]_{i j}\left(t, t_{1}\right) W_{j \beta}^{\dagger} \mathcal{G}_{\alpha \beta}\left(t_{1}, t^{\prime}, 0, x^{\prime}\right)$,

The corresponding advance component is zero. Here $1 /(i \partial / \partial t-\hat{H}(t))$ is the retarded component of the electron Green's function in the dot. This definition is different from that given in the main part of the paper, see Eq. (11). The latter will appear naturally in the end of this calculation with an additional term $\sim W^{\dagger} W$, see Eq. (13), describing escape of electrons from the dot through the leads. We represent Eq. (A.7) in the form

$$
\begin{aligned}
\mathcal{G}_{\alpha \beta}^{(K)}\left(t, t^{\prime}, x, x^{\prime}\right)= & G_{\alpha \beta}^{K}\left(t-t^{\prime}, x-x^{\prime}\right)+\int d t_{1} d t_{2} G_{\alpha \gamma}^{R}\left(t-t_{1}, x\right) \\
& \times\left[W \frac{1}{i \partial / \partial t-\hat{H}(t)} W^{\dagger}\right]_{\gamma \delta}\left(t_{1}, t_{2}\right) \mathcal{G}_{\delta \beta}^{(K)}\left(t_{2}, t^{\prime}, 0, x^{\prime}\right),
\end{aligned}
$$

take the limit $x=0$ and, using $G_{\alpha \beta}^{R}\left(t-t^{\prime}, 0\right)$ from Eq. A.3. obtain for $x^{\prime}<0$

$\mathcal{G}_{\alpha \beta}^{(K)}\left(t, t^{\prime}, 0, x^{\prime}\right)=\int d t_{1}\left[1-\hat{W} \frac{i \pi \nu}{i \partial / \partial t-\hat{H}(t)} \hat{W}^{\dagger}\right]_{\alpha \delta}^{-1}\left(t, t_{1}\right) G_{\delta \beta}^{(K)}\left(t_{1}, t^{\prime}, 0, x^{\prime}\right)$.

Substituting this expression to Eq. A.10) and taking $x=+|\delta| \rightarrow 0$, we find

$\mathcal{G}_{\alpha \beta}^{(K)}\left(t, t^{\prime},+|\delta|, x^{\prime}\right)=\int d t_{1} \mathcal{S}_{\alpha \gamma}\left(t, t_{1}\right) G_{\gamma \beta}^{K}\left(t_{1}-t^{\prime},-x^{\prime}\right), \quad x^{\prime}<0$, 
where the scattering matrix $\mathcal{S}_{\alpha \beta}\left(t, t^{\prime}\right)$ is defined by Eq. (12).

Equation A.12 is valid for $x^{\prime}<0$. We have to repeat the procedure described above to calculate the electron Green's function in the leads for $x^{\prime}>0$. Since the equations which determine evolution of the Green's function from $x^{\prime}<0$ to $x^{\prime}>0$ are conjugated to those for $x$, we obtain

$\mathcal{G}_{\alpha \beta}^{(K)}\left(t, t^{\prime},+|\delta|,+|\delta|\right)=\iint d t_{1} d t_{2} \mathcal{S}_{\alpha \gamma}\left(t, t_{1}\right) G_{\gamma \delta}^{K}\left(t_{1}-t_{2}, 0\right) \mathcal{S}_{\delta \beta}^{\dagger}\left(t_{2}, t^{\prime}\right)$.

The currents in the left (right) leads are given by

$\left\langle I_{1(\mathrm{r})}(t)\right\rangle=e v_{\mathrm{F}} \sum_{\alpha \in L(R)}\left(\mathcal{G}_{\alpha \alpha}^{(K)}(t, t,+|\delta|,+|\delta|)-\mathcal{G}_{\alpha \alpha}^{(K)}(t, t,-|\delta|,-|\delta|)\right)$,

where $\alpha=1 \ldots N_{\mathrm{l}}$ for left lead and $\alpha=N_{\mathrm{l}}+1 \ldots N_{\mathrm{ch}}$ for right lead; coordinate $\delta$ is in the lead just before the contact with the dot: $\delta>0$ and $\delta \rightarrow 0$. Function $\mathcal{G}_{\alpha \alpha}^{(K)}(t, t,-|\delta|,-|\delta|)$ is taken for incoming electrons and is given by Eq. A.5 and consequently,

$\mathcal{G}_{\alpha \alpha}^{(K)}(t, t,-|\delta|,-|\delta|)=f(+0), \quad f(t)=\int_{-\infty}^{+\infty} e^{i \omega t} \tilde{f}(\omega) \frac{d \omega}{2 \pi}$.

The total dc current from the dot should be zero $\left\langle I_{1}\right\rangle+\left\langle I_{\mathrm{r}}\right\rangle=0$ to ensure no charge accumulation on the dot, where $\left\langle I_{1(\mathrm{r})}\right\rangle=\int_{0}^{\tau_{\mathrm{o}}}\left\langle I_{1(\mathrm{r})}(t)\right\rangle d t / \tau_{\mathrm{o}}$. Therefore, we can rewrite the expression for dc current through the dot as

$I(t)=\frac{N_{\mathrm{r}}\left\langle I_{1}\right\rangle-N_{\mathrm{l}}\left\langle I_{\mathrm{r}}\right\rangle}{N_{\mathrm{ch}}}=\left\langle I_{1}\right\rangle=-\left\langle I_{\mathrm{r}}\right\rangle$.

Substituting Eqs. A.13) and A.15 into Eqs. A.14 and using Eq. A.16 we obtain Eq. (9).

\section{Appendix B.}

In this Appendix we derive Eq. (29) for the current noise correlation function through a quantum dot. The quantum mechanical operator of the current through left (right) lead is

$I_{1(\mathrm{r})}(t)=e v_{\mathrm{F}} \sum_{\alpha \in L(R)}\left(\psi_{\alpha}^{\dagger}(t,+\delta) \psi_{\alpha}(t,+\delta)-\psi_{\alpha}^{\dagger}(t,-\delta) \psi_{\alpha}(t,-\delta)\right)$

with $\psi_{\alpha}(t, \pm \delta)$ being the operator for outgoing $(+\delta)$ or incoming $(-\delta)$ electrons through channel $\alpha$, cf. to Eq. A.14.

Substituting the expression for the current operator, Eq. (B.1) into Eq. (28) and using the charge conservation in the dot on time $\tau_{\mathrm{o}} \gg 1 / \omega$, we obtain the following expression for the current correlation function (below $\delta \rightarrow 0$, but $\delta>0$ ):

$$
\begin{aligned}
S= & e^{2} v_{\mathrm{F}}^{2} \int_{0}^{\tau_{\mathrm{o}}} d t d t^{\prime}\left(\operatorname{tr}\left\{\hat{\Lambda} \hat{\mathcal{G}}^{<}\left(t^{\prime}, t,+\delta,+\delta\right) \hat{\Lambda} \hat{\mathcal{G}}^{>}\left(t, t^{\prime},+\delta,+\delta\right)\right\}\right. \\
& -\operatorname{tr}\left\{\hat{\Lambda} \hat{\mathcal{G}}^{<}\left(t^{\prime}, t,+\delta,-\delta\right) \hat{\Lambda} \hat{\mathcal{G}}^{>}\left(t, t^{\prime},-\delta,+\delta\right)\right\} \\
& -\operatorname{tr}\left\{\hat{\Lambda} \hat{\mathcal{G}}^{<}\left(t^{\prime}, t,-\delta,+\delta\right) \hat{\Lambda} \hat{\mathcal{G}}^{>}\left(t, t^{\prime},+\delta,-\delta\right)\right\} \\
& \left.+\operatorname{tr}\left\{\hat{\Lambda} \hat{\mathcal{G}}^{<}\left(t^{\prime}, t,-\delta,-\delta\right) \hat{\Lambda} \hat{\mathcal{G}}^{>}\left(t, t^{\prime},-\delta,-\delta\right)\right\}\right) .
\end{aligned}
$$


Here we introduced the electron Green's functions in the leads according to the following definitions (for a review of the Keldysh Green's function formalism see [25]):

$\mathcal{G}_{\alpha \beta}^{<}\left(t, t^{\prime}, x, x^{\prime}\right)=i\left\langle\psi_{\beta}^{\dagger}\left(t^{\prime}, x^{\prime}\right) \psi_{\alpha}(t, x)\right\rangle, \quad \mathcal{G}_{\alpha \beta}^{>}\left(t, t^{\prime}, x, x^{\prime}\right)=-i\left\langle\psi_{\alpha}(t, x) \psi_{\beta}^{\dagger}\left(t^{\prime}, x^{\prime}\right)\right\rangle$.

The Green functions $\hat{\mathcal{G}}^{<,>}$can be written in terms of the retarded, advanced and Keldysh Green's functions:

$\hat{\mathcal{G}}^{<}\left(t, t^{\prime}, x, x^{\prime}\right)=\frac{1}{2}\left(\hat{\mathcal{G}}^{(K)}\left(t, t^{\prime}, x, x^{\prime}\right)-\hat{\mathcal{G}}^{(R)}\left(t, t^{\prime}, x, x^{\prime}\right)+\hat{\mathcal{G}}^{(A)}\left(t, t^{\prime}, x, x^{\prime}\right)\right)$,

$\hat{\mathcal{G}}^{>}\left(t, t^{\prime}, x, x^{\prime}\right)=\frac{1}{2}\left(\hat{\mathcal{G}}^{(K)}\left(t, t^{\prime}, x, x^{\prime}\right)+\hat{\mathcal{G}}^{(R)}\left(t, t^{\prime}, x, x^{\prime}\right)-\hat{\mathcal{G}}^{(A)}\left(t, t^{\prime}, x, x^{\prime}\right)\right)$.

The next step is to represent the Green's functions as a product of incoming electron Green's functions, Eqs. (A.2)-A.5), and the scattering matrix, Eq. (12). The procedure is similar to one, described in Appendix A We have the following relations: $\hat{\mathcal{G}}^{(R)}\left(t, t^{\prime},-\delta,+\delta\right)=\hat{\mathcal{G}}^{(A)}\left(t, t^{\prime},+\delta,-\delta\right)=0$ $\hat{\mathcal{G}}^{(R, A)}\left(t, t^{\prime},+\delta,+\delta\right)=\hat{G}^{(R, A)}\left(t-t^{\prime}, 0\right)$,

$\hat{\mathcal{G}}^{(K)}\left(t, t^{\prime},+\delta,+\delta\right)=\int \hat{\mathcal{S}}\left(t, t_{1}\right) \hat{G}^{(K)}\left(t_{1}-t_{2}, 0\right) \hat{\mathcal{S}}^{\dagger}\left(t_{2}, t^{\prime}\right) d t_{1} d t_{2}$,

$\hat{\mathcal{G}}^{(R, K)}\left(t, t^{\prime},+\delta,-\delta\right)=\int \hat{\mathcal{S}}\left(t, t_{1}\right) \hat{G}^{(R, K)}\left(t_{1}-t^{\prime},+\delta\right) d t_{1}$,

$\hat{\mathcal{G}}^{(A, K)}\left(t, t^{\prime},-\delta,+\delta\right)=\int \hat{G}^{(A, K)}\left(t-t_{1},-\delta\right) \hat{\mathcal{S}}^{\dagger}\left(t_{1}, t^{\prime}\right) d t_{1}$.

Now the derivation of Eq. (29) reduces to simple algebraic calculations. With the help of Eqs. (B.4) and (B.5) we rewrite Eq. (B.2) in terms of the retarded, advanced and Keldysh components of the Green function. Then we represent these components as a product of scattering matrices and the Green's functions of the incoming electrons, using Eqs. (B.6)-(B.10). The result is given by Eq. (29).

[1] L. P. Kouwenhoven et al., in Electron Transport in Quantum Dots, NATO ASI conference proceedings, edited by L. P. Kouwenhoven, G. Schon, and L. L. Sohn (Kluwer, Dordrecht, 1997).

[2] A. D. Stone, Phys. Rev. Lett. 54, 2692 (1985).

[3] B. L. Altshuler, JETP Lett. 41, 648 (1985).

[4] P. A. Lee and A. D. Stone, Phys. Rev. Lett. 55, 1622 (1985).

[5] B. L. Altshuler and B. I. Shklovskii, Sov. Phys. JETP 64, 127 (1986).

[6] R. A. Webb, S. Washburn, C. P. Umbach, and R. B. Laibowitz, Phys. Rev. Lett. 54, 2696 (1985).

[7] C. W. J. Beenakker, Rev. Mod. Phys. 69, 731 (1997).

[8] B. L. Altshuler and D. E. Khmelnitskii, JETP Lett. 42, 359 (1985).

[9] A. G. Huibers and et al, Phys. Rev. Lett. 81, 200 (1998).

[10] A. G. Huibers and et al., Phys. Rev. Lett. 83, 5090 (1999).

[11] B. L. Altshuler and B. D. Simons, in Mesoscopic Quantum Physics, edited by E. Akkermans and et al (Elsevier, 1995).

[12] A. Altland, C. R. Offer, and B. D. Simons, in Disordered Systems and Quantum Chaos, edited by I. V. Lerner (Kluwer/Plenum, New York, 1999).

[13] L. P. Gorkov, A. I. Larkin, and D. E. Khmel'nitskii, JETP Lett. 30, 228 (1979).

[14] N. Argaman, Phys. Rev. Lett. 75, 2750 (1995).

[15] S. Iida, H. A. Weidenmüller, and J. A. Zuk, Phys. Rev. Lett. 64, 583 (1990).

[16] H. U. Baranger, R. A. Jalabert, and A. D. Stone, Phys. Rev. Lett. 70, 3876 (1993).

[17] H. U. Baranger, R. A. Jalabert, and A. D. Stone, Chaos 3, 665 (1993).

[18] H. U. Baranger and P. A. Mello, Phys. Rev. Lett. 73, 142 (1994).

[19] R. A. Jalabert, J. L. Pichard, and C. W. J. Beenakker, Europhys. Lett. 27, 255 (1994).

[20] R. Landauer, IBM J. Res. Dev. 1, 223 (1957). 
[21] D. S. Fisher and P. A. Lee, Phys. Rev. B 23, 6851 (1981).

[22] M. Büttikker, Phys. Rev. Lett. 57, 1761 (1986).

[23] A. D. Stone and A. Szafer, IBM J. Res. Dev. 32, 384 (1988).

[24] A. A. Abrikosov, L. P. Gorkov, and I. E.Dzyaloshinskii, Methods of Quantum Field Theory in Statistical Physics (Prentice-Hall, Englewood Cliffs, NJ, 1963).

[25] J. Rammer and H. Smith, Rev. Mod. Phys. 58, 323 (1986).

[26] H. U. Baranger, A. D. Stone, and D. P. Divincenzo, Phys. Rev. B 37, 6521 (1988).

[27] H. U. Baranger and A. D. Stone, Phys. Rev. B 40, 8169 (1989).

[28] R. Blümel and U. Smilansky, Phys. Rev. Lett. 60, 477 (1988).

[29] M. L. Mehta, Random Matrices (Academic Press, Boston, 1991).

[30] J. J. M. Verbaarschot, H. A. Weidenmüller, and M. R. Zirnbauer, Phys. Reports 129, 367 (1985).

[31] C. H. Lewenkopf and H. A. Weidenmüller, Ann. Phys. (N. Y.) 212, 53 (1991).

[32] P. W. Brouwer, Phys. Rev. B 51, 16878 (1995).

[33] Y. V. Fyodorov and H.-J. Sommers, J. Math. Phys. 38, 1918 (1997).

[34] K. B. Efetov, Sov. Phys. JETP 56, 467 (1982).

[35] K. B. Efetov, Adv. Physics 32, 53 (1983).

[36] K. B. Efetov, Supersymmetry in Disorder and Chaos (Cambridge University Press, New York, 1997).

[37] B. A. Muzykantskii and D. Khmelnitskii, JETP Lett. 62, 68 (1995).

[38] A. V. Andreev, O. Agam, B. D. Simons, and B. L. Altshuler, Phys. Rev. Lett. 76, 3947 (1996).

[39] B. L. Altshuler, A. G. Aronov, D. E. Khmelnitskii, and A. I. Larkin, Quantum Theory of Solids (Mir publisher, Moscow, 1982).

[40] H. U. Baranger and P. A. Mello, Phys. Rev. B 51, 4703 (1995).

[41] P. W. Brouwer and C. W. J. Beenakker, Phys. Rev. B 51, 7739 (1995).

[42] E. McCann and I. V. Lerner, Phys. Rev. B 57, 7219 (1998).

[43] U. Sivan, Y. Imry, and A. G. Aronov, Europhys. Lett. 28, 115 (1994).

[44] B. L. Altshuler, Y. Gefen, A. Kamenev, and L. S. Levitov, Phys. Rev. Lett. 78, 2803 (1997).

[45] M. G. Vavilov and I. L. Aleiner, Phys. Rev. B 60, R16311 (1999).

[46] M. G. Vavilov and I. L. Aleiner, Phys. Rev. B 64, 085115 (2001).

[47] M. L. Polianski and P. W. Brouwer, J. Phys. A: Math. Gen. 36, 3215 (2003).

[48] M. Büttiker, A. Pretre, and H. Thomas, Phys. Rev. Lett. 70, 4114 (1993).

[49] M. Büttiker, H. Thomas, and A. Pretre, Z. Phys. B 94, 133 (1994).

[50] X. B. Wang and V. E. Kravtsov, Phys. Rev. B 64, 033313 (2001).

[51] V. I. Yudson, E. Kanzieper, and V. E. Kravtsov, Phys. Rev. B 64, 045310 (2001).

[52] D. J. Thouless, Phys. Rev. B 27, 6083 (1983).

[53] Q. Niu, Phys. Rev. Lett. 64, 1812 (1990).

[54] D. V. Averin and K. K. Likharev, in Mesoscopic Phenomena in Solids, edited by B. L. Altshuler, P. A. Lee, and R. A. Webb (Elsevier, Amsterdam, 1991).

[55] L. P. Kouwenhoven et al., Phys. Rev. Lett. 67, 1626 (1991).

[56] I. L. Aleiner and A. V. Andreev, Phys. Rev. Lett. 81, 1286 (1998).

[57] P. W. Brouwer, Phys. Rev. B 58, R10135 (1998).

[58] F. Zhou, B. Spivak, and B. L. Altshuler, Phys. Rev. Lett. 82, 608 (1999).

[59] T. A. Shutenko, I. L. Aleiner, and B. L. Altshuler, Phys. Rev. B 61, 10366 (2000).

[60] M. Blaauboer and E. J. Heller, Phys. Rev. B 64, 241301 (2001).

[61] M. G. Vavilov, V. Ambegaokar, and I. L. Aleiner, Phys. Rev. B 63, 195313 (2001).

[62] B. Wang, J. Wang, and H. Guo, Phys. Rev. B 68, 155326 (2003).

[63] O. Entin-Wohlman, A. Aharony, and Y. Levinson, Phys. Rev. B 65, 195411 (2002).

[64] V. I. Belinicher and B. I. Sturman, Sov. Phys. Usp. 23, 199 (1980).

[65] V. I. Fal'ko and D. E. Khmelnitskii, Sov. Phys. JETP 68, 186 (1989).

[66] A. G. Aronov and V. E. Kravtsov, Phys. Rev. B 47, 13409 (1993).

[67] V. E. Kravtsov and V. I. Yudson, Phys. Rev. Lett. 70, 210 (1993).

[68] S. Fishman, D. R. Grempel, and R. E. Prange, Phys. Rev. Lett. 49, 509 (1982).

[69] D. M. Basko, M. A. Skvortsov, and V. E. Kravtsov, Phys. Rev. Lett. 90, 096801 (2003).

[70] D. M. Basko and V. E. Kravtsov, Phys. Rev. B 71, 085311 (2005).

[71] A. V. Andreev and A. Kamenev, Phys. Rev. Lett. 85, 1294 (2000).

[72] L. S. Levitov, cond-mat/0103617

[73] A. V. Andreev and E. G. Mishchenko, Phys. Rev. B 64, 233316 (2001).

[74] L. S. Levitov and G. B. Lesovik, JETP Lett. 58, 230 (1993).

[75] D. A. Ivanov, H. Lee, and L. S. Levitov, Phys. Rev. B 56, 6839 (1997).

[76] M. Moskalets and M. Büttiker, Phys. Rev. B 66, 035306 (2002). 
[77] M. L. Polianski, M. G. Vavilov, and P. W. Brouwer, Phys. Rev. B 65, 245314 (2002).

[78] A. A. Bykov, G. M. Gusev, and Z. D. Kvon, Sov. Phys. JETP 70, 742 (1990).

[79] H. Linke et al., Europhys. Lett. 44, 341 (1998).

[80] M. Switkes, C. M. Marcus, K. Campman, and A. C. Gossard, Science 283, 1905 (1999).

[81] P. W. Brouwer, Phys. Rev. B 63, R121303 (2001).

[82] L. DiCarlo, C. M. Marcus, and J. S. Harris, Phys. Rev. Lett. 91, 246804 (2003).

[83] M. G. Vavilov, L. DiCarlo, and C. M. Marcus, Phys. Rev. B 71, 241309 (2005).

[84] P. W. Brouwer and I. L. Aleiner, Phys. Rev. Lett. 82, 390 (1999).

[85] I. L. Aleiner, P. W. Brouwer, and L. Glazman, Phys. Reports 358, 309 (2002).

[86] D. S. Golubev and A. D. Zaikin, Phys. Rev. B 69, 075318 (2004).

[87] P. W. Brouwer, A. Lamacraft, and K. Flensberg, Phys. Rev. Lett. 94, 136801 (2005).

[88] M. Buttiker and M. L. Polianski, cond-mat/0508220 (2005).

[89] P. W. Brouwer et al., Phys. Rev. Lett. 79, 913 (1997).

[90] H. Nyquist, Phys. Rev. 32, 110 (1928).

[91] J. B. Johnson, Phys. Rev. 32, 97 (1928).

[92] M. Moskalets and M. Büttiker, Phys. Rev. B 66, 035303 (2002).

[93] O. Agam, I. L. Aleiner, and A. I. Larkin, Phys. Rev. Lett. 85, 3153 (2000).

[94] A. Lamacraft, Phys. Rev. Lett. 91, 036804 (2003).

[95] K. B. Efetov, Phys. Rev. Lett. 74, 2299 (1995).

[96] B. D. Simons and B. L. Altshuler, Phys. Rev. Lett. 70, 4063 (1993).

[97] B. L. Altshuler, A. G. Aronov, A. I. Larkin, and D. E. Khmelnitskii, Sov. Phys. JETP 54, 411 (1981).

[98] P. W. Brouwer and C. W. J. Beenakker, J. Math. Phys. 37, 4904 (1996).

[99] L. Onsager, Phys. Rev. 38, 2265 (1931).

[100] A. V. Shytov, Phys. Rev. B 71, 085301 (2005).

[101] P. W. Brouwer, A. Lamacraft, and K. Flensberg, Phys. Rev. B 72, 075316 (2005).

[102] M. Moskalets and M. Büttiker, Phys. Rev. B 64, 201305(R) (2001).

[103] J. N. H. J. Cremers and P. W. Brouwer, Phys. Rev. B 65, 115333 (2002).

[104] E. R. Mucciolo, C. Chamon, and C. M. Marcus, Phys. Rev. Lett. 89, 146802 (2002).

[105] S. K. Watson, R. M. Potok, C. M. Marcus, and V. Umansky, Phys. Rev. Lett. 91, 258301 (2003).

[106] M. Blaauboer, Phys. Rev. B 65, 235318 (2002).

[107] J. Wang and B. Wang, Phys. Rev. B 65, 153311 (2002). 